\title{
Magnetic resonance imaging of the shoulder
}

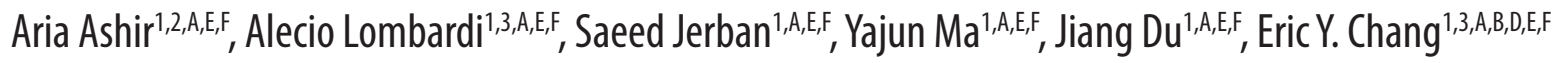 \\ 'University of California, San Diego, USA \\ ${ }^{2}$ Drexel University College of Medicine, USA \\ ${ }^{3}$ VA San Diego Healthcare System, USA
}

\section{Abstract}

\begin{abstract}
The aim of this article is to review the use of magnetic resonance imaging (MRI) for the evaluation of shoulder pain, which is a common clinical complaint of the musculoskeletal system. MRI is an essential auxiliary tool to evaluate these patients because of its high resolution and high sensitivity in depicting the soft tissues. This article will review the imaging technique, normal imaging anatomy, and most common imaging findings of disorders of tendons, labrum, and ligaments of the shoulder. It will also discuss common systemic diseases that manifest in the shoulder as well as disorders of the acromioclavicular joint and bursae. New advances and research in MRI have provided additional potential uses for evaluating shoulder derangements.
\end{abstract}

Key words: MRI, shoulder, glenohumeral joint, acromioclavicular (AC) joint, rotator cuff.

\section{Introduction}

Shoulder pain is one of the most common musculoskeletal complaints. Studies have shown an annual incidence of 14.7 per 1000 patients and a lifetime prevalence up to $70 \%$ $[1,2]$. Shoulder pain affects work productivity, activities of daily life, and quality of life. Internal derangements of the shoulder including diseases of the muscles and tendons, joints, and labroligamentous and periarticular structures are common causes of shoulder pain. Diagnostic imaging has become increasingly important for the evaluation of these structures. Magnetic resonance imaging (MRI) is a crucial diagnostic tool with its multiplanar imaging, high resolution, and lack of ionising radiation. MR arthrography is a more sensitive tool for the evaluation of the labroligamentous structures, joint capsule and cartilage, and additional signs of rotator cuff tears. Already the preferred imaging modality for evaluating many internal derangements of the shoulder, advances in MRI have provided additional potential benefits for evaluating shoulder pathology.

This paper will review MRI techniques for evaluating the shoulder, normal shoulder anatomy and MRI appear- ance, and MRI findings of specific disorders of the rotator cuff tendons and muscles, the glenohumeral joint and labrum, the acromioclavicular (AC) joint, and the shoulder bursae. Common injuries, diagnostic pitfalls, and recent advances in MRI of the shoulder will be reviewed.

\section{Technique}

The patient is imaged in the supine position with their affected arm at the side. A local shoulder coil is required in order to optimize the signal-to-noise ratio. The shoulder should be held in partial external rotation. Internal rotation should be avoided because this can distort the appearance of the supraspinatus and subscapularis tendons [3]. Excessive external rotation should also be avoided because potential fluid in the biceps tendon sheath may simulate a small tear of the leading edge of the supraspinatus tendon due to partial volume effects [3].

Images are obtained in the axial, coronal oblique, and sagittal oblique planes (Figure 1). The axial slices are perpendicular to the long axis of the body and serve as a localiser for the other two planes. The coronal oblique plane is obtained perpendicular to the glenoid face, typi-

Correspondence address:

Dr. Eric Y. Chang, Radiology Service, VA San Diego Healthcare System, San Diego, USA, e-mail: ericchangmd@gmail.com

Authors' contribution:

A Study design · B Data collection · C Statistical analysis · D Data interpretation - E Manuscript preparation · F Literature search · G Funds collection 

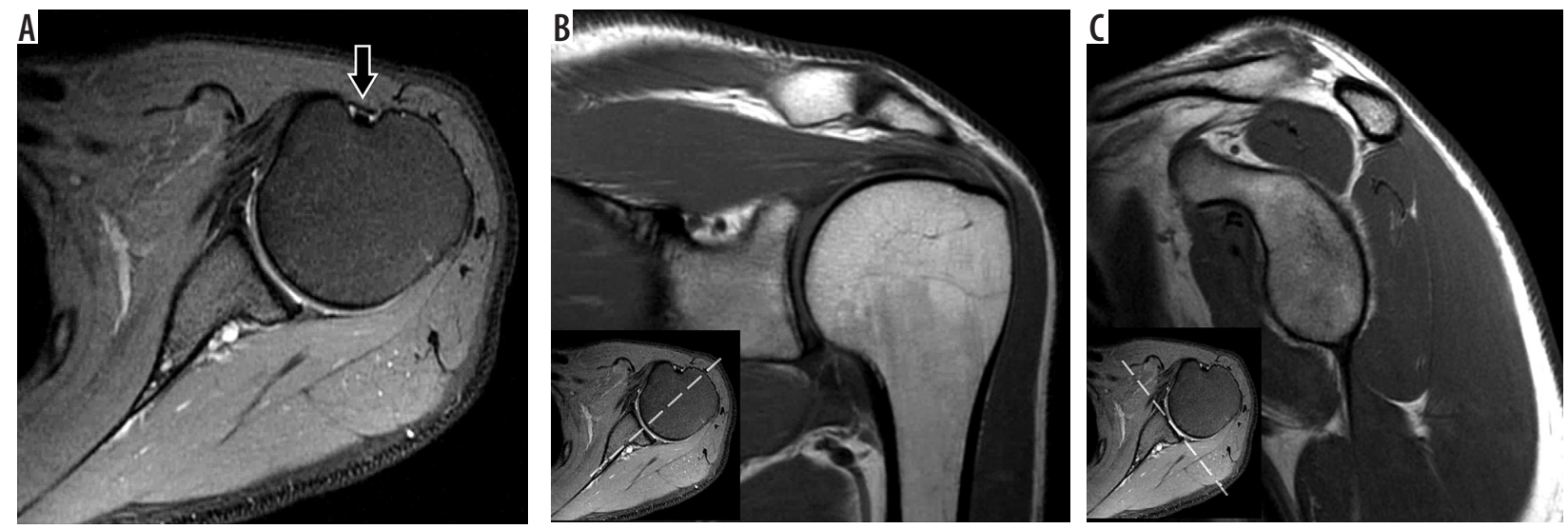

Figure 1. Shoulder magnetic resonance imaging planes. A) Axial proton density-weighted fat-suppressed as well as (B) coronal oblique and (C) sagittal oblique T1-weighted magnetic resonance images of the shoulder show the typical positioning of the glenohumeral joint during the exam. The shoulder is in partial external rotation as seen by the position of the long head of the biceps tendon on the axial images (arrow). Correlation between axial, coronal oblique, and sagittal oblique planes are showed (inset images in B and C)

cally parallel to the supraspinatus muscle and tendon, and should cover the region from the infraspinatus to the subscapularis muscles. The sagittal oblique plane is perpendicular to the coronal oblique, parallel to the glenoid face, and should cover from the scapular neck medially through the greater tuberosity laterally. The axial plane images can help evaluate the subscapularis tendon, glenohumeral joint, and the glenoid labrum. The sagittal and coronal oblique images can be helpful in evaluating the labrum, biceps tendon, AC joint, rotator interval, supraspinatus, and infraspinatus muscles/tendons.

On 1.5 and 3T units, the smallest field of view (FOV) that does not result in aliasing should be used, typically $13-15 \mathrm{~cm}$ [4]. A sample protocol for imaging at $3 \mathrm{~T}$ is shown in Table 1. Many standard shoulder protocols will include the following fast spin echo sequences: axial proton density (PD)-weighted, coronal and sagittal oblique T1-weighted, and coronal and sagittal oblique T2-weighted with fat suppression sequences. Fluid-sensitive (PD-weighted or T2-weighted) sequences are helpful in evaluating the rotator cuff. T1-weighted sequences will help with evaluating muscle pathology, haemorrhage, and marrow abnormalities $[4,5]$.

MR arthrography assists with evaluation of shoulder instability and some cases of RCT pathology, such as partial-thickness and subscapularis tears. MR arthrography is typically performed via a direct intra-articular injection using a dilute gadolinium-containing contrast solution. Although intra-articular injection of gadolinium-containing contrast agents is not Food and Drug Administration approved, it is routinely performed as an "off-label" technique. With recent concerns regarding the safety of gadolinium-containing contrast agents, it is comforting to know that studies have not shown significant intracranial gadolinium deposition following MR arthrography [6,7]. A variety of anterior- and posterior-based approaches may be used utilising fluoroscopic or ultrasound guidance. At the most typically used field strengths (1.5-3T), the optimum gadolinium concentration for maximal signalto-noise ratio ranges from 0.7 to $3.4 \mathrm{mmol} / \mathrm{l}$ (or less than $2 \mathrm{mmol} / \mathrm{l}$ if iodinated contrast used also used) [8,9]. Generally, a T1-weighted sequence with/without fat suppression is used alongside a fluid-sensitive sequence that can detect pathology that is not communicating with the joint. The addition of fat suppression to T1-weighted sequences may help with the diagnosis of RCT tears [4].

\section{General shoulder anatomy}

The shoulder is comprised of two articulations: the glenohumeral joint and the AC joint. The glenohumeral joint is an articulation between the hemispheric head of the humerus and the glenoid cavity of the scapula.

Table 1. Sample non-contrast shoulder imaging protocol at $3 \mathrm{~T}^{\mathrm{a}}$

\begin{tabular}{|l|c|c|c|c|c|}
\hline Sequence & TR $(\mathrm{ms})$ & TE $(\mathrm{ms})$ & $\begin{array}{c}\text { Slice thickness } \\
(\mathbf{m m})\end{array}$ & FOV (mm) & Matrix \\
\hline Axial PD FS & 3000 & 30 & 3.0 & 130 & $320 \times 288$ \\
\hline Coronal oblique T1 & 1000 & 10 & 3.0 & 130 & $288 \times 256$ \\
\hline Coronal oblique T2 FS & 3500 & 70 & 3.0 & 130 & $320 \times 288$ \\
\hline Sagittal oblique T1 & 600 & 10 & 3.0 & 130 & $288 \times 256$ \\
\hline Sagittal oblique T2 FS & 3500 & 70 & 3.0 & 130 & $320 \times 288$ \\
\hline
\end{tabular}

FOV - field of view, FS - fat-suppressed, PD - proton density, TE - echo time, TR - repetition time, ${ }^{\text {a }}$ - refer to Figure 1 for imaging plane examples 
The glenohumeral articulation is stabilised by the joint capsule, the glenohumeral ligaments, rotator cuff muscles, and the labrum. The labrum is a fibrocartilage ring that is adherent to the glenoid. This structure helps increase the stability of the glenohumeral articulation by creating a deeper and more concave socket on the glenoid. The labrum consists of the anterior, posterior, superior, and inferior portions. The superior portion of the labrum and supraglenoid tubercle serve as the anchors for the long head of the biceps tendon. There are multiple normal labral variants that will be discussed in the glenohumeral joint and labrum section. The glenohumeral joint capsule may insert on the periphery of the labrum or on the adjacent scapula, and distally it will insert on the anatomic neck of the humerus.

The glenohumeral ligaments include the superior, middle, and inferior glenohumeral ligaments. Each ligament extends from the anterior margin of the scapula, near the labrum, to the anatomic neck of the humerus. The inferior glenohumeral ligament complex has anterior and posterior bands and an intervening axillary pouch that typically attach to the anterior inferior and posterior inferior labrum, respectively. There is significant variation in the number and size of the glenohumeral ligaments among individuals [10].

The rotator cuff is composed of tendons from the supraspinatus, infraspinatus, teres minor, and subscapularis muscles. The supraspinatus, infraspinatus, and teres minor muscles originate from the posterior surface of the scapula and insert on the greater tuberosity of the humerus. The supraspinatus inserts the most superiorly, followed by the infraspinatus and then the teres minor. The supraspinatus and teres minor have one muscle belly each, while the infraspinatus has several. In some individuals, the infraspinatus and teres minor tendons may be fused [11]. The subscapularis originates from the anterior surface of the scapula, contains multiple muscle bellies, and insert on the lesser tuberosity of the humerus. The transverse humeral ligament passes from the lesser to the greater tuberosity of the humerus, and some fibres of the subscapularis tendon join the transverse humeral ligament. The long head of the biceps tendon passes under this ligament.

The rotator interval is a triangular space between the supraspinatus and subscapularis tendons and the base of the coracoid process. It has a capsule covering it, which is mostly composed of tissue from the coracohumeral ligament. This space houses the superior glenohumeral ligament and the tendon of the long head of the biceps. The coracohumeral and superior glenohumeral ligaments contribute to the biceps pulley, which helps to keep the long head of the biceps from displacing.

The superior rotator cuff tendons course under the coracoacromial arch and AC joint and then continue to insert on the greater tuberosity of the humerus. The coracoacromial arch is formed by the coracoid process anteriorly, the acromion posteriorly, and is roofed by the coracoacromial ligament.

The shoulder has several bursae that help reduce friction between tendon-bone and tendon-tendon interfaces. These include the subacromial-subdeltoid (SA-SD) bursa, subscapular recess or bursa, subcoracoid bursa, coracoclavicular bursa, and supra-acromial bursa. The glenohumeral joint communicates with the subscapular recess. Between the supraspinatus tendon and the coracoacromial arch is the SA-SD bursa. The SA-SD and subcoracoid bursae communicate in $11-50 \%$ of patients [12].

The AC joint is a synovial joint between the acromion and distal clavicle, and it has a fibrous capsule that is reinforced by the fibres of the AC ligament and trapezius muscle. The coracoacromial and coracoclavicular ligaments also help maintain the AC joint.

\section{Rotator cuff and muscles}

Rotator cuff tendon (RCT) disease has been found to be the most common aetiology of shoulder pain, accounting for nearly $70 \%$ of shoulder pain visits $[13,14]$. RCT pathology can be due to tears, inflammation, and degeneration. Although imaging modalities like radiographs, ultrasound, and computed tomography (CT) have indications for evaluating the shoulder, MRI is the gold standard according to the American College of Radiology (ACR) appropriateness criteria for assessing both traumatic and atraumatic shoulder pain in suspected rotator cuff disease $[15,16]$. While radiographs provide an important initial study and ultrasound may be helpful in detecting fullthickness tears for evaluating the shoulder, MRI provides excellent, comprehensive imaging of soft-tissue structures, specifically the rotator cuff and labrum, and is thus the exam of choice. MR arthrography may be indicated in certain rotator cuff tears and is particularly helpful for diagnosing partial-thickness, articular-sided, supraspinatus tears [15].

Rotator cuff tendinopathy can be classified as tendon degeneration or inflammation and is thought to be caused by intrinsic factors like tendon insult or overload, or extrinsic factors like impingement by surrounding structures. Rotator cuff tendinopathy can cause significant pain and has an incidence of $0.3-5.5 \%$ and an annual prevalence of $0.5-7.4 \%$ [17]. Signs of tendinopathy on MRI include increased signal within the tendon that has lower intensity than that of fluid on T2-weighted sequences. There may be tendon thickening, thinning, or fraying, which is the observation of a hypointense signal at the tendon margin that becomes indistinct, but there is an absence of gaps within tendon fibres (Figure 2).

When evaluating the RCT for tears, the supraspinatus and infraspinatus are the most frequently affected, and they are best evaluated in the coronal oblique plane. The subscapularis is best evaluated in the axial plane and the teres minor in the sagittal oblique plane. It is impor- 


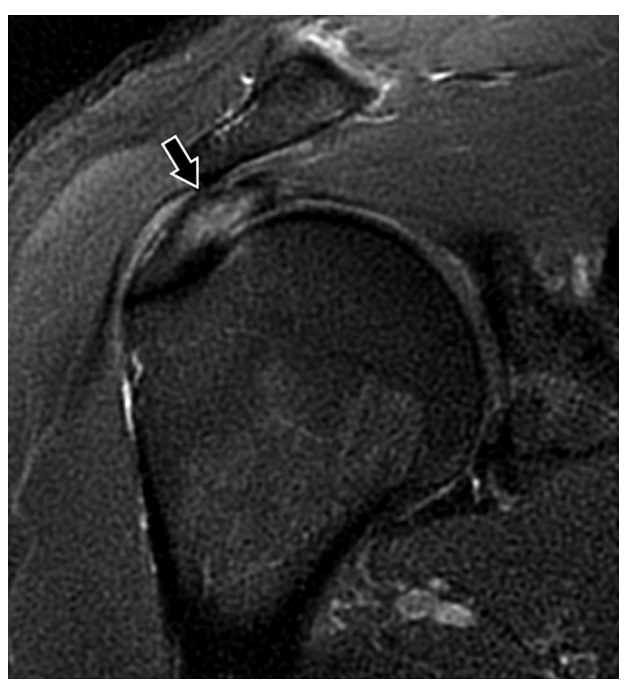

Figure 2. Tendinosis in a 45-year-old man with unilateral shoulder pain. Coronal proton density-weighted, fat-suppressed magnetic resonance image shows increased signal in the superior rotator cuff (arrow) without fluid gap, consistent with tendinopathy

tant, however, to assess all imaging planes because some small tears may be detected only on another plane. Evaluation of non-fat suppressed and fat-suppressed images should be made side-by-side, and initial evaluation using the coronal oblique plane can be performed to look for large cuff tears and anatomical distortions. T2-weighted images with fat suppression are highly sensitive for pathology but may be prone to artifacts, and therefore any abnormality seen using a fat-suppressed sequence should be confirmed in all three planes of the study. The axial images can be viewed with focus on the subscapularis. The T1-weighted sequence is useful for evaluating muscle atrophy and hypertrophic changes in the coracoacromial arch that may be causing mechanical impingement.
The typical normal appearance of healthy RCT varies but should not approach fluid unless there is a tear. Most portions of the healthy tendon are hypointense on all pulse sequences, although the lateral portion may demonstrate intermediate signal intensity, which has been attributed to the magic angle effect $[18,19]$. Normal rotator cuff muscles show intermediate signal intensity on all pulse sequences $[20,21]$.

MRI is used to evaluate both full-thickness and partial-thickness tears. Full-thickness rotator cuff tears extend from the articular surface to the bursal surface of the cuff. The prevalence of full-thickness tears in cadaveric samples has been reported to be 5 to $25 \%$ [10]. A recent study of elderly symptomatic individuals who never sought care found a $30 \%$ prevalence of full-thickness tears on imaging [22]. Sharma et al. reviewed shoulder MRI exams and reported a sensitivity of $89.6 \%$ for detecting fullthickness tears and $100 \%$ for partial-thickness tears, and a specificity of $100 \%$ for full-thickness tears and $86.6 \%$ for partial-thickness tears when compared with arthroscopy [23]. The MRI findings of a full-thickness tear include a focal discontinuity in the tendon extending from the articular to the bursal side, seen as a fluid signal intensity on T2-weighted images. However, hypointense or isointense signal may be seen in the tendon if there is chronic scarring, as may also be found in tendons after repair. Fluid intensity signal may be found in the SA-SD bursa and in the joint space (Figure 3). There are secondary signs of full-thickness tears, which include tendon retraction, superior translation of the humeral head, and muscle atrophy. The myotendinous junction is usually below the AC joint, so a proximal displacement of this junction may signal a full-thickness tear. Careful evaluation of tendon retraction and muscle atrophy on MRI is important since
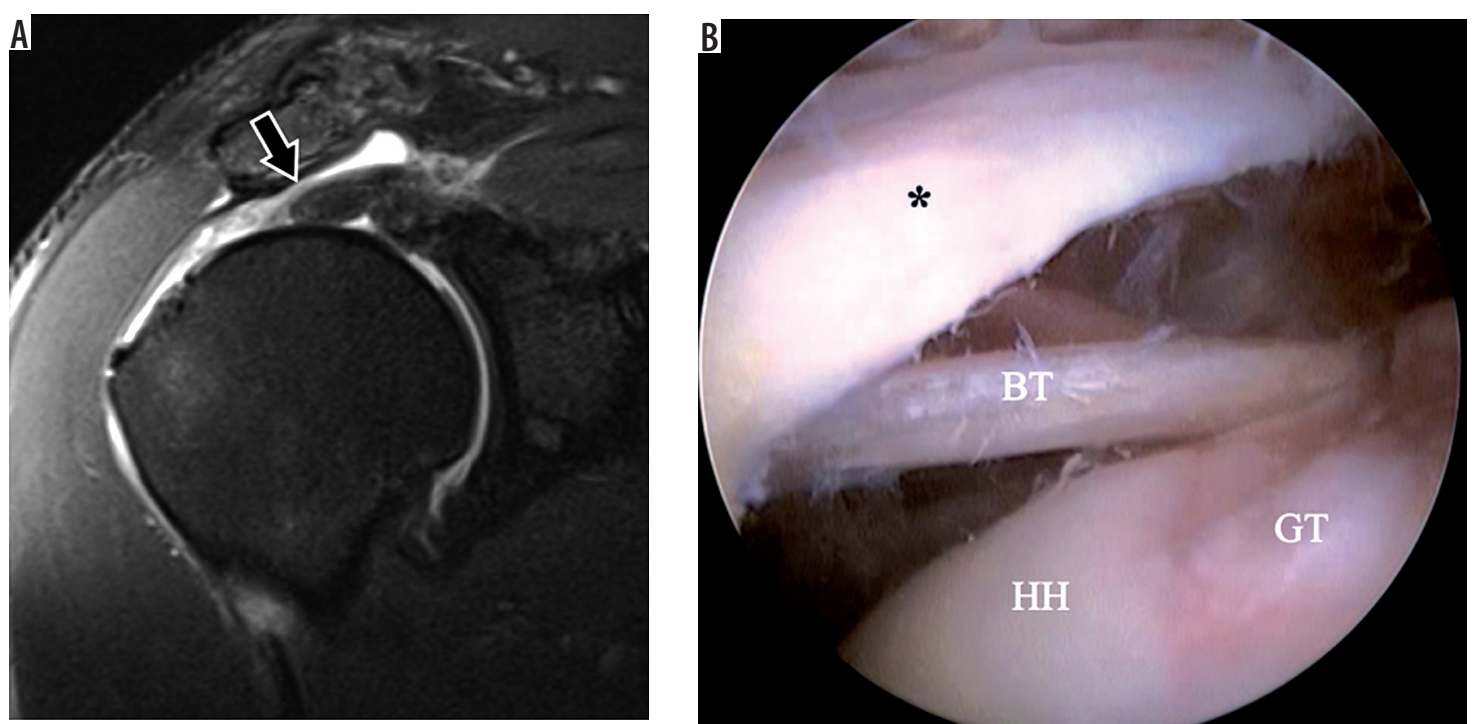

Figure 3. Full-thickness cuff tear in a 50-year-old man with shoulder pain. A) Coronal oblique, proton density-weighted, fat-suppressed magnetic resonance image depicts a full-thickness tear of the superior cuff with retraction to the mid-humeral head (arrows). B) Arthroscopic image with scope in the subacromial-subdeltoid bursa shows the lateral bursal-side of the cuff (asterisk) with a direct intra-articular view of the humeral head ( $\mathrm{HH}$ ), long head of the biceps tendon (BT), and bare greater tuberosity (GT) 

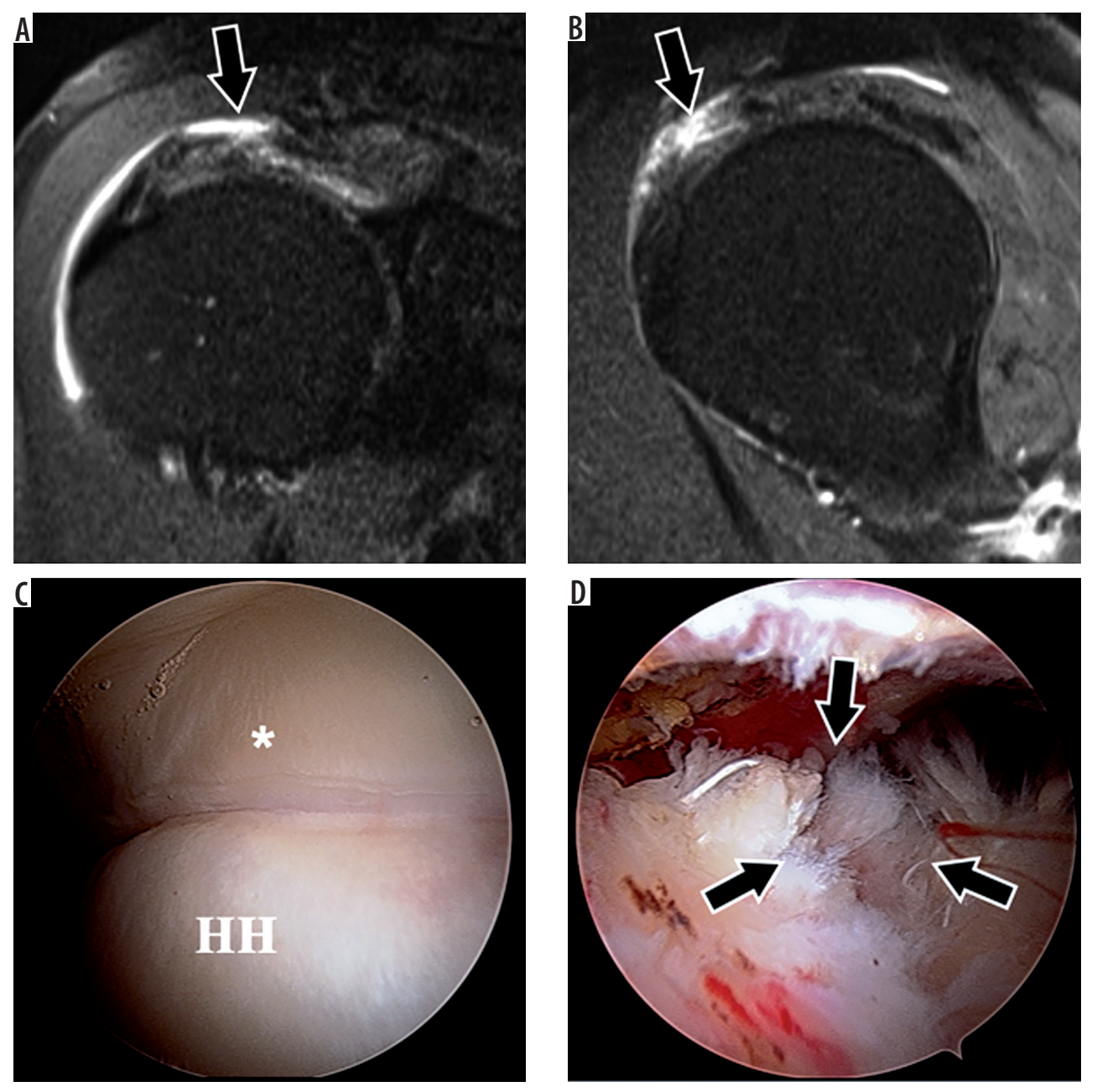

Figure 4. High-grade partial-thickness bursal-sided tear in a 53-year-old woman. A) Coronal oblique and B) sagittal oblique proton density-weighted fat-suppressed magnetic resonance images show a large bursal-sided tear (arrows) near the critical zone with medial delamination, associated with subacromial-subdeltoid bursitis. C) An intact articular-sided cuff was seen at arthroscopy (asterisk). D) Image with scope in the bursa confirms very high-grade bursal sided tear (arrows). $\mathrm{HH}$ - humeral head

it can define the approach to surgical repair. The degree of retraction can also provide information about the prognosis. The anterior-posterior dimensions of the tear can be analysed on the sagittal oblique plane. Multiple scoring systems based on cross-sectional imaging exist for evaluating rotator cuff muscle quality, such as the Goutallier (assessing fatty infiltration) and Warner (assessing atrophy) classifications. Zanetti et al. introduced the supraspinatus tangent sign, which is assessed on sagittal oblique images at the level of the scapula where the body, spine, and coracoid intersect [24]. If the supraspinatus muscle belly does not extend above the line connecting the tip of the coracoid to the superior aspect of the scapular spine, the tangent sign is positive, indicating atrophy. Recently, the supraspinatus tangent sign was found to be a prognostic indicator for operative outcomes in patients with full-thickness tears, with higher reliably than the Warner and Goutallier classifications [25].

Partial-thickness tears are incomplete tears that only involve the articular or bursal side of the cuff. Articular-sided

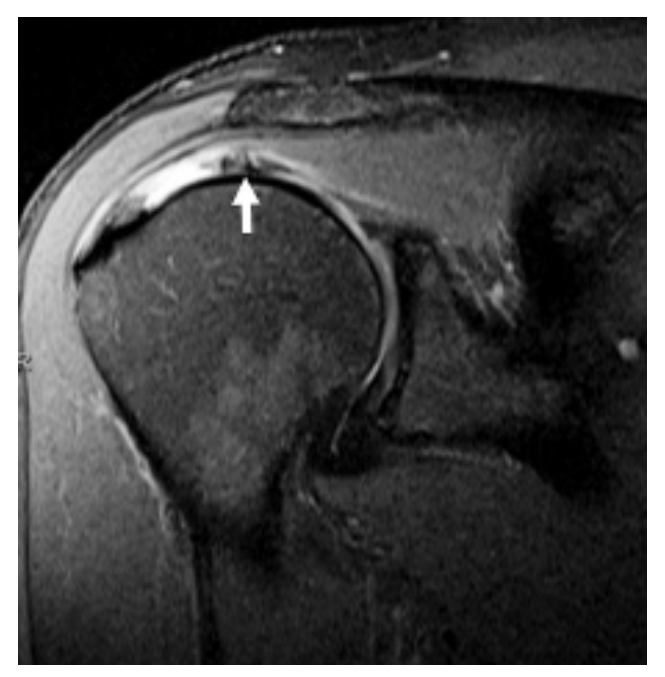

Figure 5. Accentuated rotator cable due to a supraspinatus tear involving the crescent in a 41-year-old man. Sagittal proton density-weighted fat-suppressed magnetic resonance image shows a high-grade, partial-thickness articular-sided tear of the supraspinatus tendon with retraction, resulting in a thickened appearance of the rotator cable (arrow) 

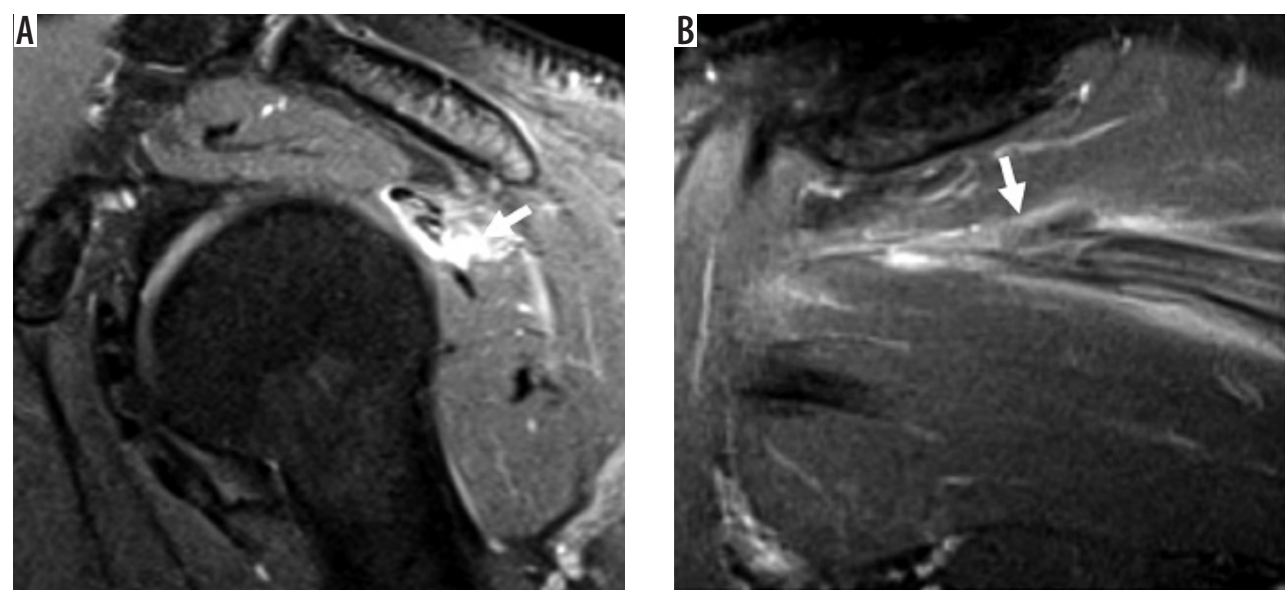

Figure 6. Delaminating tear of the infraspinatus tendon with differential retraction in a right shoulder of a 46-year-old man. A) Sagittal oblique and B) coronal oblique proton density-weighted, fat-suppressed magnetic resonance images show a tear of the infraspinatus predominantly involving the transverse portion of the layered tendon with retraction and surrounding muscular oedema (arrows)
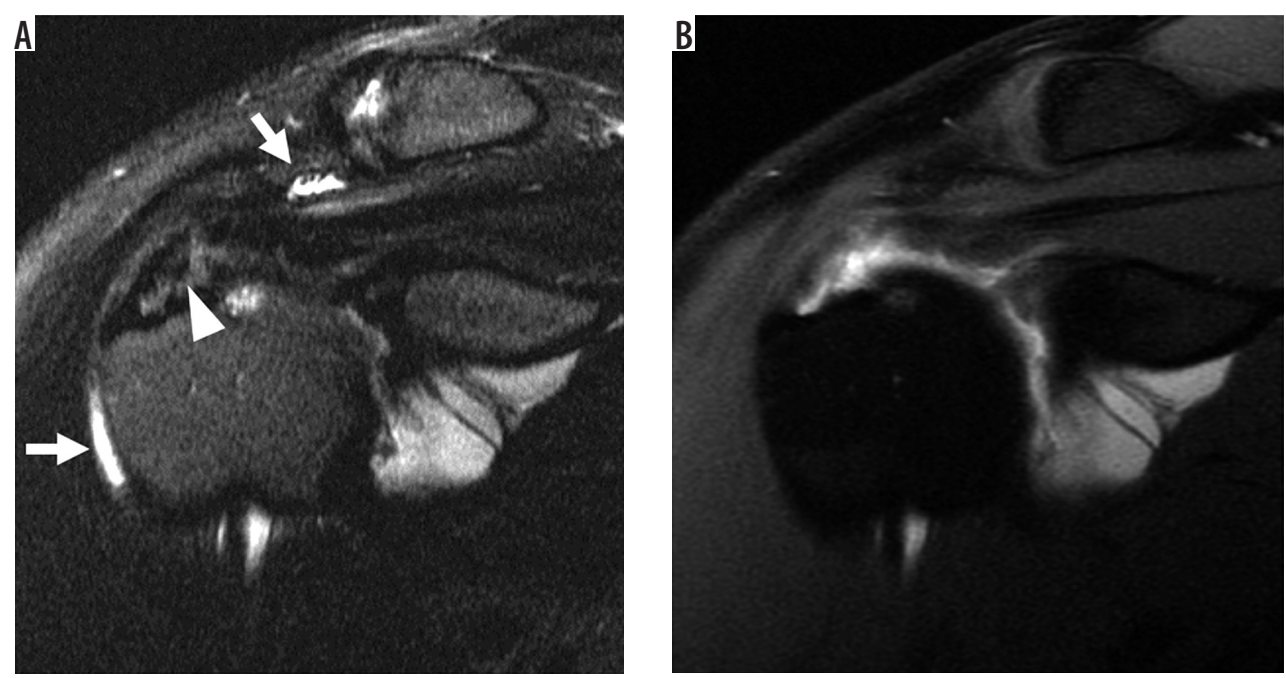

Figure 7. Magnetic resonance (MR) arthrography demonstrating a high-grade, partial-thickness, articular-sided tear in a 30-year-old professional baseball player. A) Coronal oblique T2-weighted, fat-suppressed MR image shows a very high-grade tear of the supraspinatus tendon near the footprint (arrowhead) with a small amount of fluid in the subacromial-subdeltoid (SA-SD) bursa (arrows). B) Coronal oblique, T1-weighted, fat-suppressed MR arthrogram image shows an absence of fluid in the SA-SD bursa, confirming that the tear is partial-thickness rather than full-thickness
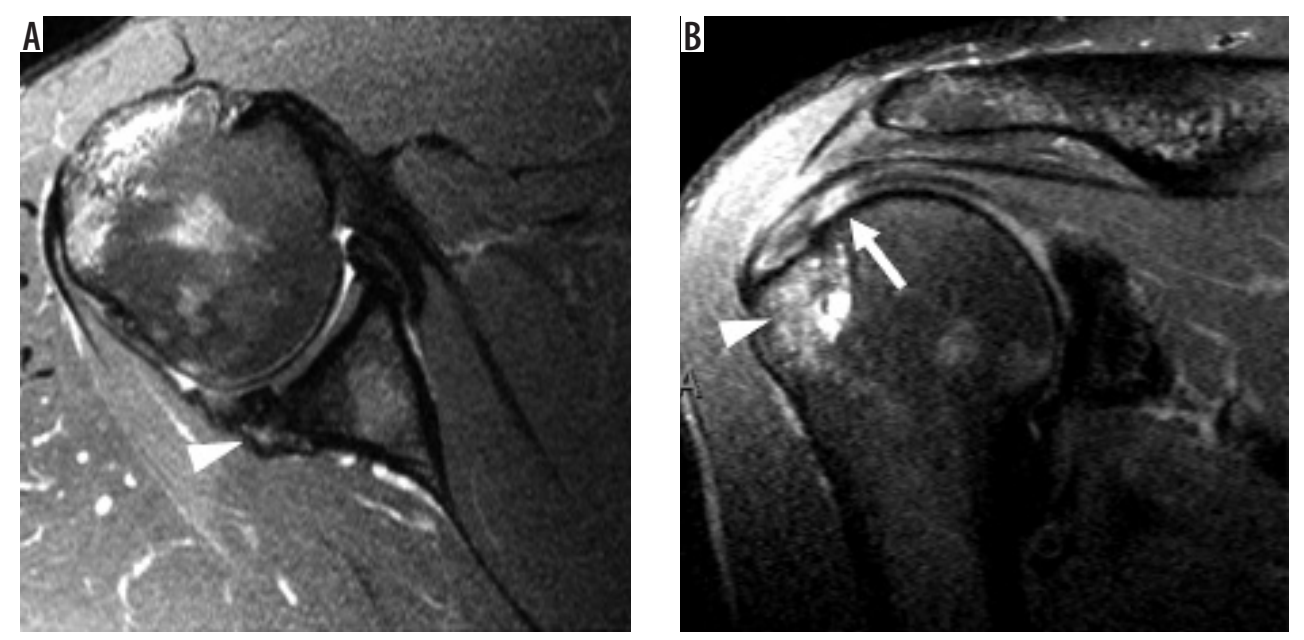

Figure 8. Internal impingement in a 30-year-old professional baseball pitcher with painful throwing. A) Axial T2-weighted, fat-suppressed magnetic resonance image shows posterior labral tear (arrow) and bone marrow oedema-like changes (arrowhead). B) Coronal oblique T2-weighted, fat-suppressed image show partial thickness articular-sided tear of the posterosuperior cuff (arrow) and bone marrow oedema-like changes (arrowhead) 

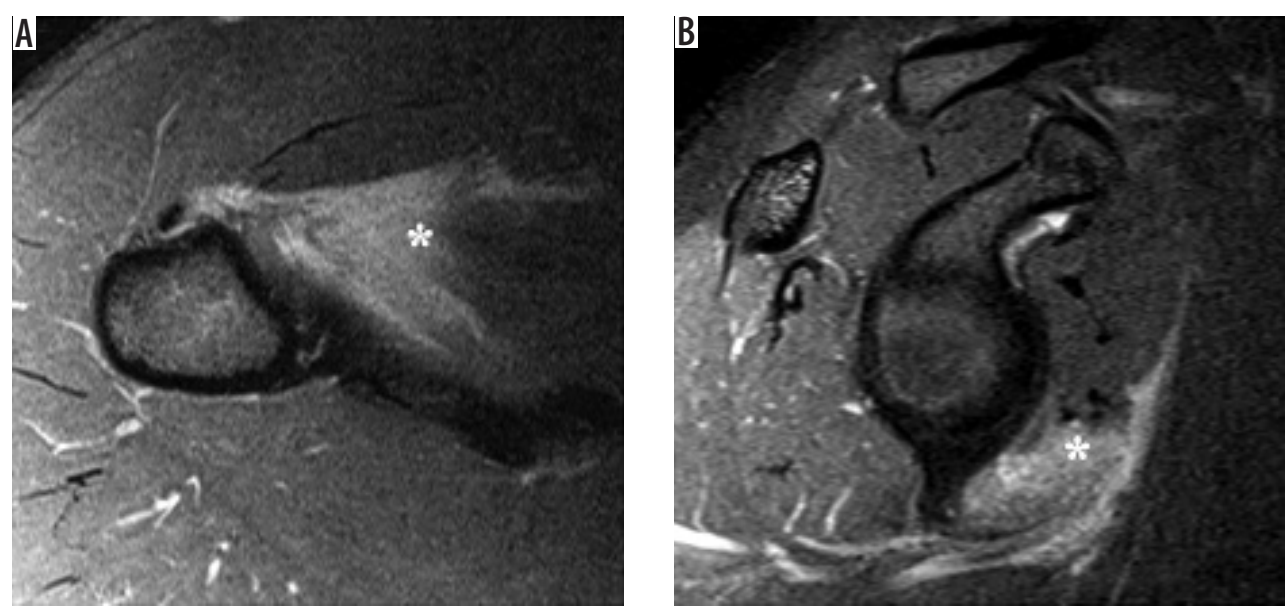

Figure 9. Inferior subscapularis myotendinous junction strain in a 22-year-old pitcher with acute posterior shoulder pain. A) Axial and B) sagittal oblique, proton density-weighted, fat-suppressed magnetic resonance images show oedema centred around the myotendinous junction of the inferior subscapularis muscle (asterisk), consistent with a grade 2 strain

tears are more common than bursal-sided tears. A study on athletes showed a similar trend, but noted a higher number of articular-sided tears in athletes who throw repeatedly, while bursal-sided tears were likely in weightlifters [26]. Differences in the composition of these two regions (complex of tendon, joint, and ligament in articular-sided v. tendon bundles in bursal-sided regions) may be a reason why articular-sided regions are more vulnerable to a tensile load [27]. The appearance of partial-thickness tears on MRI is focal discontinuity of the tendon with high signal intensity on T2-weighted images. There may be findings of surface fraying and changes in tendon calibre. Fat suppression sequences can help display the tendon defect. Some high-grade partial-thickness bursal-sided tears may be mistaken for full-thickness tears. In these cases, searching for a prominent subdeltoid or SA-SD bursal fluid distension with a relative paucity of effusion in the glenohumeral joint (disproportionate fluid sign), can aid diagnosis because this finding is much more likely in bursal-sided tears [28] (Figure 4). Partial-thickness articular-sided tears can also involve the rotator cable or crescent. The rotator cable is a fibrous, $\mathrm{U}$-shaped structure, that runs perpendicular to the supraspinatus and infraspinatus tendons. This layer of the rotator cuff and glenohumeral joint capsule is thought to reduce the stress applied to the rotator crescent, which is the thin, avascular region lateral to the cable [29] (Figure 5). Tears involving the rotator cable are thought to cause more symptoms and lead to recurrent tears, and should be addressed with early repair [30]. These tears may explain why some overhead athletes continue to show little improvement when treated with debridement only and no repair [31].

Intrasubstance tears are a type of incomplete tear only within the substance of the rotator cuff and do not extend to the surface. It can be important to identify this type of tear on imaging because it can be missed during arthroscopy. Delaminated tears where there is a horizontal split of the tendon substance are thought to be associated with poorer surgical outcomes; however, a study by Kwon et al. noted that these tears may represent a confounding rather than prognostic factor because the arthroscopic findings are seen in elderly patients with chronic degenerative tears of long symptom duration alongside findings like muscle atrophy, poor tendon quality, and larger tear sizes [32,33]. A notable injury is a delaminating tear of the infraspinatus tendon with differential retraction of the layered tendon, previously referred to as a "novel lesion" [34] (Figure 6). These lesions can cause significant pain and weakness on external rotation. Although these findings may be seen on MRI, they can be missed on arthroscopy or bursoscopy [35], adding to its clinical relevance.

MR arthrography can be a useful tool when there is suspicion of an occult cuff tear. Although studies have shown similar diagnostic value when non-contrast MRI and MR arthrography are used to evaluate bursal-sided tears, MR arthrography may have higher sensitivity in evaluating certain types of tears, such as partial-thickness articular-sided tears $[15,36]$. The main drawback is the potential patient discomfort caused by contrast injection. In partial-thickness articular surface tears, the contrast can be seen in the articular substance of the tear, while in bursal-sided tears there will be no communication with the joint space and thus no contrast visualised. In fullthickness tears the joint space will communicate with the overlying SA-SD bursa, and therefore contrast material will be found in this area (Figure 7). If contrast is seen within the substance of the subscapularis tendon, there is a possibility that it may have been introduced during injection if an anterior approach was utilised. Gadolinium contrast is used during MR arthrography; however, saline arthrography may be considered as an alternative because it is found to be equivalent in diagnosing some labral and rotator cuff pathologies, with the ability to obtain additional information from fluid sensitive sequences and reduce the cost associated with gadolinium injection [37].

Impingement syndromes were first described as conditions where there is entrapment of musculoskeletal 
structures in the shoulder and subsequent pain. Subacromial and subcoracoid impingements involve the supraspinatus and subscapularis tendons, respectively.

Subacromial impingement is the most common impingement syndrome and is due to attrition between the coracoacromial arch and the supraspinatus tendon. Additionally, attrition of the rotator cuff is thought to contribute to glenohumeral joint imbalance, superior migration of the humeral head, and narrowing of the subacromial space $[38,39]$. Although radiographs and ultrasound can be helpful in evaluating shoulder impingement, MRI gives detailed information about the extra-articular findings that can be a cause or result of shoulder impingement [16]. Acromial morphology can predispose to impingement. The Bigliani classification describes acromial morphology as follows: type I, flat; type II, curved; and type III, a hooked acromion [40]. Vanarthos et al. later described a type IV acromion with a convex undersurface [41]. One of the MRI findings that has been associated with subacromial impingement is the type III acromion. Other commonly associated findings with subacromial impingement are a lateral down-sloping acromion, an os acromiale (unfused accessory ossification centre of the acromion), osteophytes and enthesophytes extruding from the AC joint, and SA-SD bursitis. However, there has been controversy over whether these findings could really cause rotator cuff disease or impingement syndrome [40,42].

Subcoracoid impingement is a less common disease in which there is a reduction in the space of the coracohumeral interval (space between the tip of coracoid and humerus), usually defined as less than $6 \mathrm{~mm}$ [40]. This narrowing may be due to a congenitally long coracoid process, trauma, or rotator cuff repair. The affected structures can include the subscapularis tendon and the long head of the biceps tendon. There may be signs of tendinopathy or a tear in the subscapularis tendon, although this is a rare cause of subscapularis tendon tears when compared to aetiologies like anterior shoulder dislocation or degeneration associated with supraspinatus and infraspinatus pathology [21].
Internal impingement is due to repetitive contact of the rotator cuff between the greater tuberosity of the humerus and the posterosuperior glenoid, commonly seen in overhead throwing athletes like baseball players due to the repeated "cocking", where there is abduction and external rotation (ABER) of the arm [43]. Common MRI findings include posterosuperior cuff and labral tears and remodelling of the posterior humeral head that can show bone marrow oedema, subcortical cysts formation, and cortical flattening and depression (Figure 8). A potential pitfall is interpreting posterior humeral head remodelling as a Hill-Sachs lesion. A clue to the correct diagnosis lies in searching for posterior humeral head remodelling in internal impingement versus the posterosuperior humeral head remodelling found in Hill-Sachs lesions. Findings of internal impingement are seen more inferiorly than Hill-Sachs lesions on axial images, usually near the largest diameter of the humeral head [44]. Another unique injury in overhead throwing athletes is an inferior subscapularis myotendinous junction strain, for which MRI can be particularly helpful because the clinical picture can be confusing due to the possibility of symptoms manifesting posteriorly [45] (Figure 9).

Calcium hydroxyapatite crystal deposition disease, also known as calcific tendinitis, is most frequent about the shoulder and is characterised by the presence of calcium crystal deposits in the periarticular soft tissues [46], especially the tendons. Hydroxyapatite can migrate to adjacent tissues such as bursae, bones [47], and towards the myotendinous junction [48] according to the evolution stage of the disease. Hypointense linear or globular signal associated with surrounding soft tissue oedema are the most common MRI findings (Figure 10).

When evaluating the rotator cuff, it is important to consider some limitations of MR imaging. The appearance of calcium on MR imaging can be bright or dark and can sometimes be misinterpreted as tears or subacromial bone spurs. This is another reason why radiographs are an important adjunct study, because they can help elucidate some of these findings and additional bony changes. In addition,
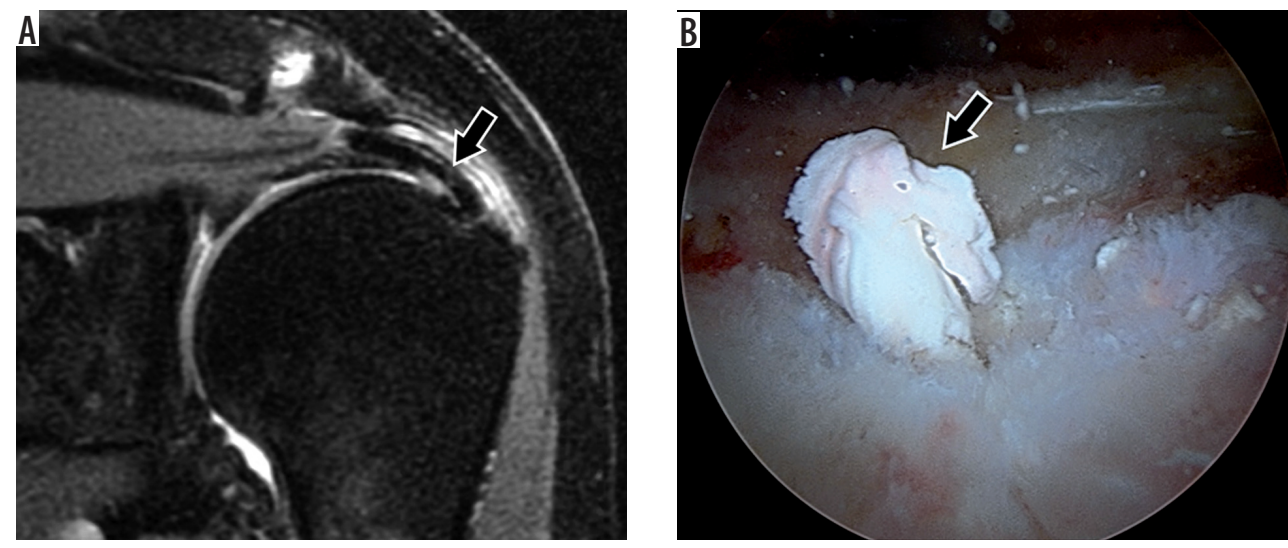

Figure 10. Calcium hydroxyapatite crystal deposition in the superior cuff in a 67-year-old woman. A) Coronal oblique, T2-weighted, fat-suppressed magnetic resonance image shows a large, hypointense deposit in the supraspinatus tendon with surrounding oedema (arrow). B) Arthroscopic image shows the bursal surface of the rotator cuff during expression of the calcium hydroxyapatite crystals from the cuff tendon 
artifacts like magic angle phenomenon or volume averaging can be significant limitations. When evaluating the rotator cuff tendons for signs of increased signal intensity on images with short echo times (i.e. PD, T1-weighted, gradient echo sequences), the magic angle phenomenon may cause a falsely increased signal due to the orientation of the tendon to the magnetic field. The anisotropic nature of tendons makes them susceptible to changes in orientation relative to the magnetic field [19]. At approximately $54.78^{\circ}$ relative to the main magnetic field, this effect becomes more prominent and can show increased signal intensity even in healthy tendons. The artifactually lengthened $\mathrm{T} 2$ remains much less than the T2 of fluid, however, and therefore exclusion of a tear can be made on longer TE sequences (greater than 60-90 ms) where only fluid remains bright. Another potential solution to distinguish between cuff tendons that are normal and degenerated is through the use of quantitative ultrashort echo time (UTE) sequences, especially magnetisation transfer parameters, which have been shown to be magic angle insensitive but remain capable of detecting aberrant changes tendons and articular cartilage [49-51].

In a recent study, 7T MRI was applied to a patient cohort for the first time to assess rotator cuff pathology. The study showed promise for the use of 7T MRI in the clinical setting to obtain high resolution rotator cuff images. However, in its current state there was an overestimation of pathology when 7T imaging was compared to arthroscopic analysis and $1.5 \mathrm{~T}$ and $3 \mathrm{~T}$ images, possibly due to artifacts like the magic angle effect and the bias of signal alteration over-interpretation [52].

\section{Glenohumeral joint/labrum}

\section{Normal anatomy}

The glenoid labrum is a fibrocartilaginous structure around the glenoid rim, which functions to increase the glenoid surface articular area and increase contact between the humeral head and the glenoid, contributing to shoulder stability [53]. Its normal appearance in the MRI should be round or triangular. The superior and inferior portions of the labrum are well-visualised on coronal oblique images, whereas the axial plane helps evaluate the anterior and posterior portions. The glenoid labrum can be described by segments (superior, anterosuperior, anterior, anteroinferior, inferior, posteroinferior, posterior, and posterosuperior) or by the clock face (12 oclock superior, 3 o'clock anterior, 6 o'clock inferior, 9 o'clock posterior). Of note, some orthopaedic surgeons may consider the clock face description of the left shoulder the opposite from the right shoulder (i.e. 3 oclock being posterior and 9 o'clock being anterior). The radiologist should be aware of this possibility and should always include a full description of the involved segments, especially when using the clock face.

Multiple findings may represent a labral tear, including an irregular line of fluid, intraarticular contrast tracking into the labral substance, or contrast between the labrum and glenoid articular cartilage. If a labral lesion extends through the joint capsule, it may form a paralabral cyst. A cyst located along the posterosuperior glenoid rim (Figure 11) may extend into the suprascapular and spinoglenoid notches, sometimes causing suprascapular nerve compression. A cyst located along the anteroinferior glenoid rim, on the other hand, may compress the axillary nerve.

\section{Anatomical variants}

Evaluating the labrum can be difficult because there are normal anatomical variants that may be mistaken for pathology. The anterior and superior portions of the labrum are usually more triangular as opposed to the more rounded posterior and inferior portions. The superior
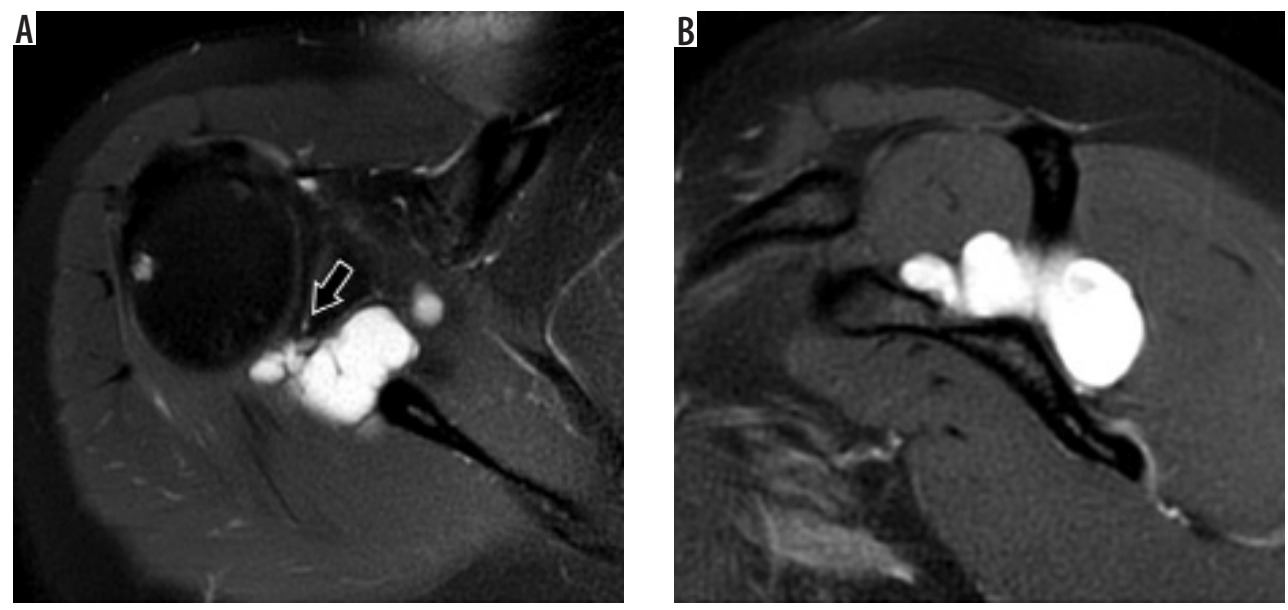

Figure 11. Paralabral ganglion cyst. T2-weighted, fat-suppressed magnetic resonance images in the A axial and B sagittal oblique planes show a posterosuperior labral tear (arrow) with a lobulated paralabral ganglion cyst extending through the suprascapular and spinoglenoid notches. No muscle signal alteration is present to suggest suprascapular neuropathy 
portion is commonly larger than the inferior, and the posterior is larger than the anterior. The superior labrum can also demonstrate a variety of appearances, including a triangular, meniscoid, or bumper shape, in decreasing order of frequency [54] (Figure 12). Additional deviations of the usual pattern (e.g. attenuated, separated, or absent) can make accurate diagnosis difficult. Classic variants include a sublabral recess or sulcus (partially unattached superior labrum, at 12 o'clock, most common), or sublabral foramen (unattached anterosuperior labrum, at 2 oclock, seen in up to $11 \%$ of individuals) (Figure 13). The Buford variant is the least common (absent anterosuperior labrum at 2 o'clock with thick middle glenohumeral ligament, seen in $1.5-2 \%$ of individuals) [55] (Figure 14). The sublabral recess is characterised by contrast or fluid interposed between the labrum and glenoid, curving superomedially towards the head of the patient. This line should not extend posterior to the biceps anchor, nor should it demonstrate irregular margins, or else a superior labral tear may be present. The sublabral foramen may show a high signal between the labrum and glenoid, while the Buford variant may show an absent or diminutive labrum.

\section{Glenohumeral instability}

The glenohumeral joint is the most commonly dislocated joint in the body. Glenohumeral dislocation may result from trauma and be unidirectional (either anterior or posterior), with anterior dislocations contributing to $95 \%$ of the cases. The incidence of anterior instability is up to 26.2 per 100,000 persons in the general population, being more common in males and athletes. The risk of recurrent destabilisation is quite high, with reports of a $39-67 \%$ rate of recurrence due to pathological changes in the joint [56].

During an anterior shoulder dislocation, the forced abduction, external rotation, and extension frequently results in structural damage. The humeral head will be displaced anteriorly, inferiorly, and medially. This injury mechanism can lead to a constellation of findings including damage to the inferior glenohumeral ligament, a Hill-Sachs lesion (posterolateral humeral head osteochondral impaction fracture), a Bankart lesion (various injuries to the anteroinferior aspect of the labrum), and/or bony Bankart lesion (fracture of anteroinferior glenoid). The loss of stabilisation from the glenohumeral ligament can lead to an incompetent joint. Delayed MRI greater than six months from the time of initial shoulder dislocation has been shown to lead to a higher incidence and severity of intra-articular pathology [57].

Radiographs may be helpful in diagnosing shoulder dislocations, particularly with knowledge of signs that assist with more difficult to diagnose posterior dislocations, such as the trough line (vertical line of bone seen at the edge of an impaction fracture at the anterior superomedial aspect of the humeral head) and the "veil of obscuration", which is seen on the AP radiographs and represents the frequently comminuted fracture of the lesser tuberosity projecting over the humeral head or glenohumeral joint creating a "veil" of bone density that may obscure the underlying posterior dislocation [58] (Figure 15). However, CT and MRI are generally more sensitive for subtle glenoid rim fractures and soft tissue damage to the labroligamentous structures, respectively. Non-contrast MRI has been found to be comparable to $\mathrm{CT}$ in evaluating glenohumeral bone loss and superior to CT, but inferior to MR arthrography in assessing labroligamentous pathology [15].

Similar to CT, MRI can be used to quantify humeral head and glenoid bone loss, aiding decision-making in surgical management $[59,60]$. It has been shown that higher humeral bone loss compared to the glenoid bone loss may indicate the need for bone augmentation in addition to labroligamentous repair for a better postoperative outcome. Newer MRI techniques such as zero echo time (ZTE) or inversion-recovery (IR) UTE imaging can be used to generate high-contrast
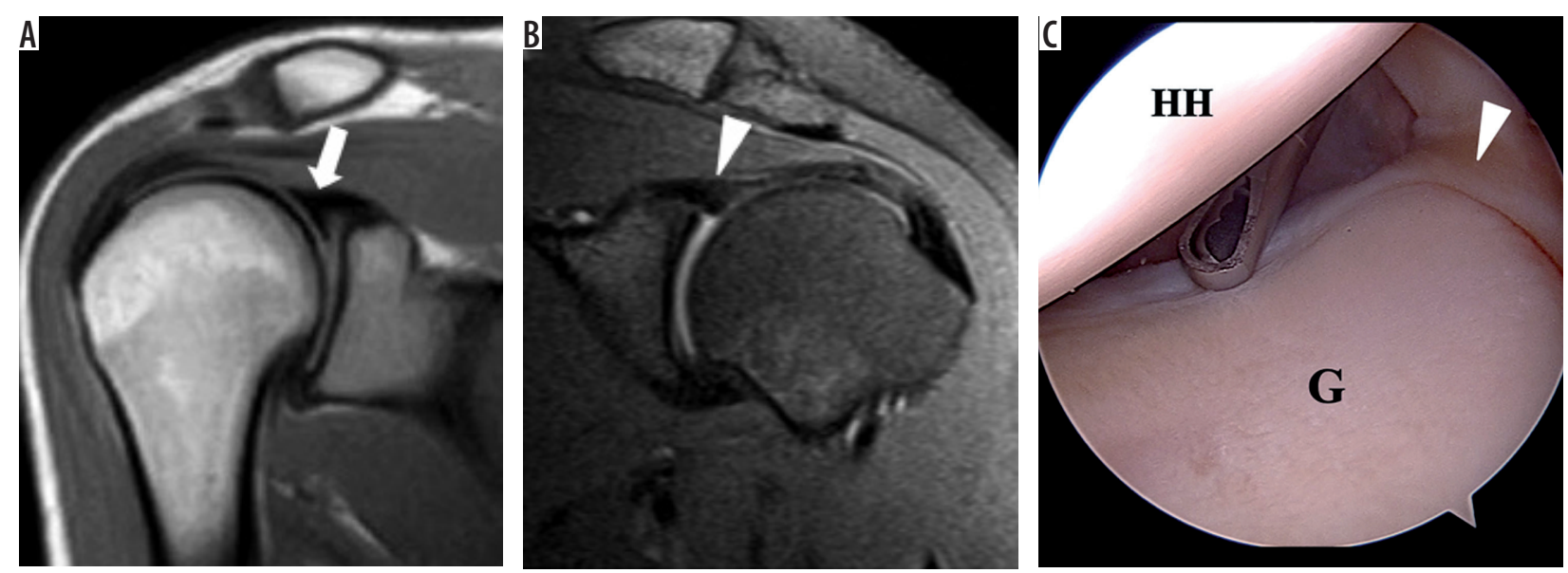

Figure 12. Superior labral variations in a 28-year-old woman (A) and a 42-year-old woman (B, C). A) Coronal oblique, T1-weighted magnetic resonance (MR) image shows a meniscoid-type superior labrum. B) Coronal oblique, proton density-weighted, fat-suppressed MR and C) arthroscopic images show an intact bumper-type labrum (arrowheads). $\mathrm{G}$ - glenoid, $\mathrm{HH}$ - humeral head 

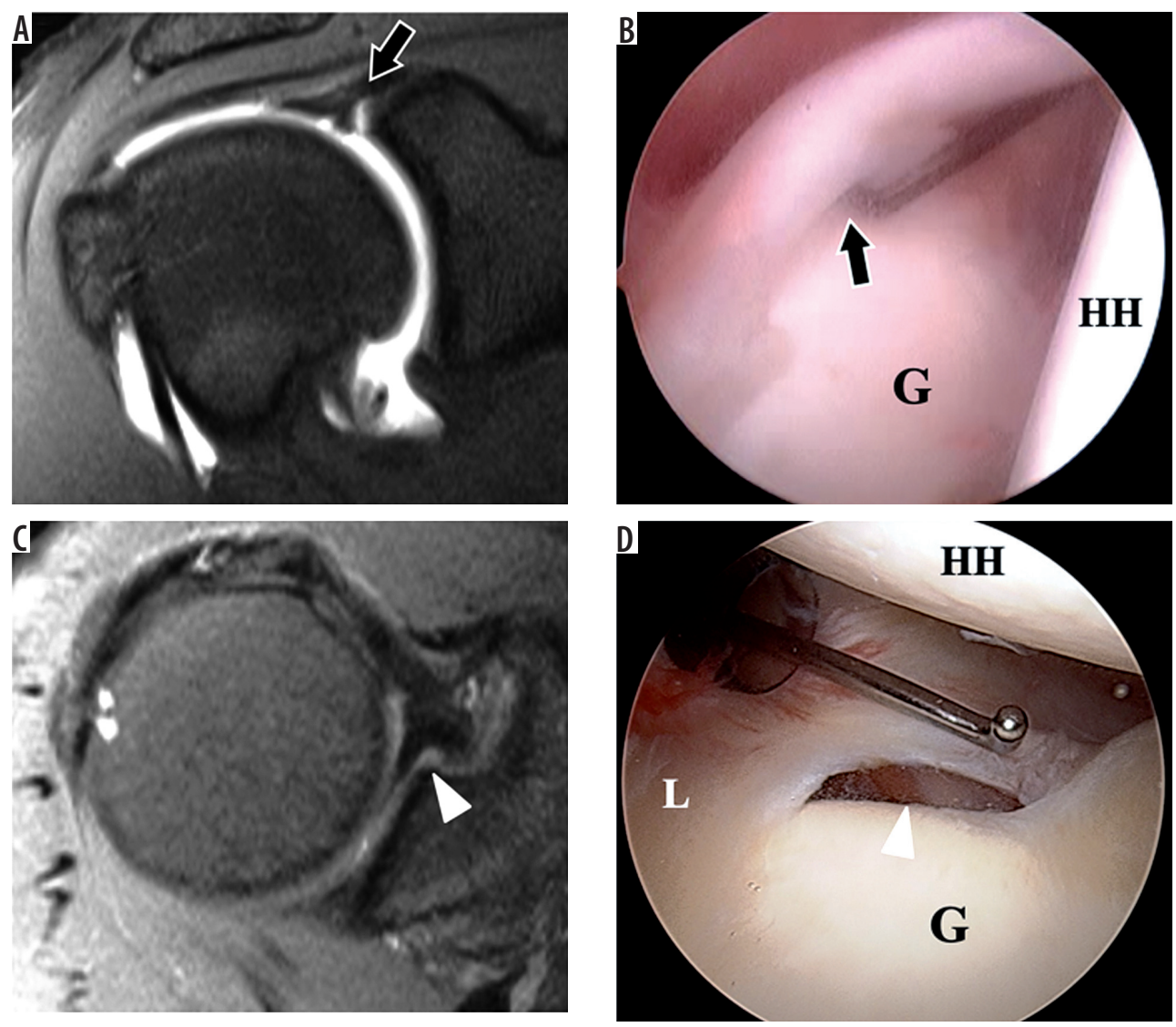

Figure 13. Labral variations in an 18-year-old man (A, B) and 48-year-old woman (C, D). A) Coronal oblique, T1-weighted, fat-sup pressed magnetic resonance (MR) arthrography image shows a sublabral recess (arrow). B) At arthroscopy, the superior labrum was stable to probing and a small recess was confirmed (arrow). C) Axial proton density-weighted, fat-suppressed MR image shows a sublabral foramen (arrowhead). D) Arthroscopic image shows a meniscoid-type superior labrum $(\mathrm{L})$ and a sublabral foramen (arrowhead) at the level of the glenoid notch. $\mathrm{G}$ - glenoid, $\mathrm{HH}$ - humeral head
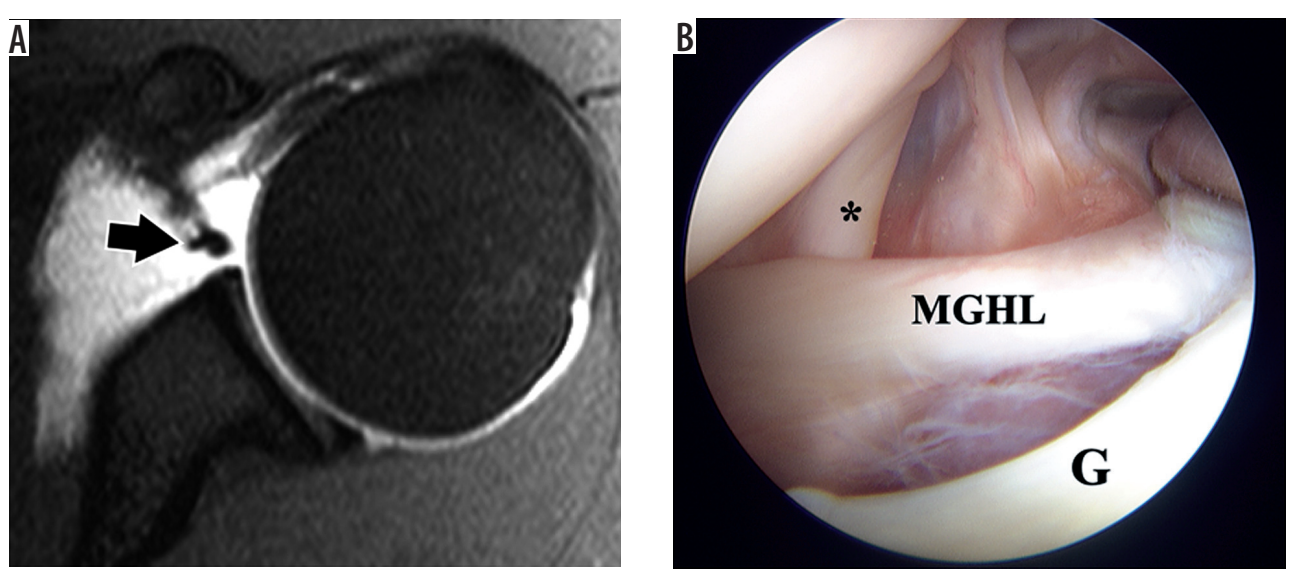

Figure 14. Buford variant in a 22-year-old man. A) Axial T2-weighted, fat-suppressed magnetic resonance image shows an absent anterosuperior glenoid labrum and thickened middle glenohumeral ligament (MGHL) (arrow). B) Arthroscopic image confirms the absent labrum along the anterosuperior glenoid (G) and thickened MGHL deep to the subscapularis tendon (asterisk)

bone images [61,62]. ZTE provides an isotropic dataset, which facilitates the creation of 3D-volume rendered images, but IR-UTE provides better suppression of non-bone tissues (Figure 16). When assessing Hill-Sachs lesions, it is essential to remember that below the coracoid there is a natural posterolateral flattening of the humeral head, also known as a pseudo-Hill-Sachs lesion. Evaluation should focus on a region of flattening or wedge-shaped defect (potential bone marrow oedema acutely) of the posterolateral humeral head, above the level of the coracoid [63].

MR arthrography can be helpful for the evaluation of subacute and chronic Bankart lesions as well as Bankart variants such as the anterior labroligamentous periosteal sleeve avulsion (ALPSA), anteroinferior labral detachment with intact periosteum (Perthes), and glenolabral articular disruption (GLAD), which represents an antero- 

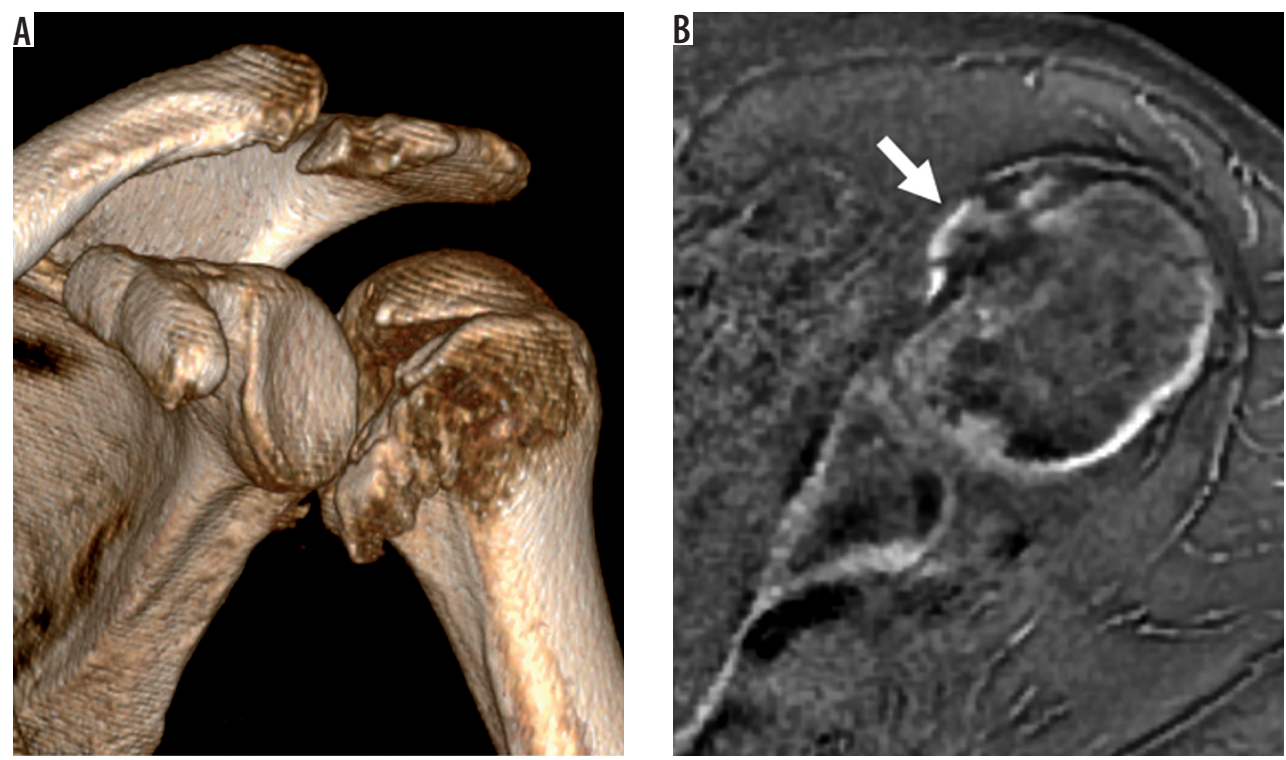

Figure 15. Seizure-induced posterior shoulder dislocation in a 42-year-old man. A) 3D volume-rendered image from computed tomography exam shows a posterior dislocation with comminuted fracture of the proximal humerus. B) Axial ZTE magnetic resonance image post-reduction confirms normal glenohumeral alignment and the comminuted fracture of the proximal humerus (arrow). The lesser tuberosity fragments have been termed the "veil of obscuration", which is a helpful radiographic sign of posterior dislocation
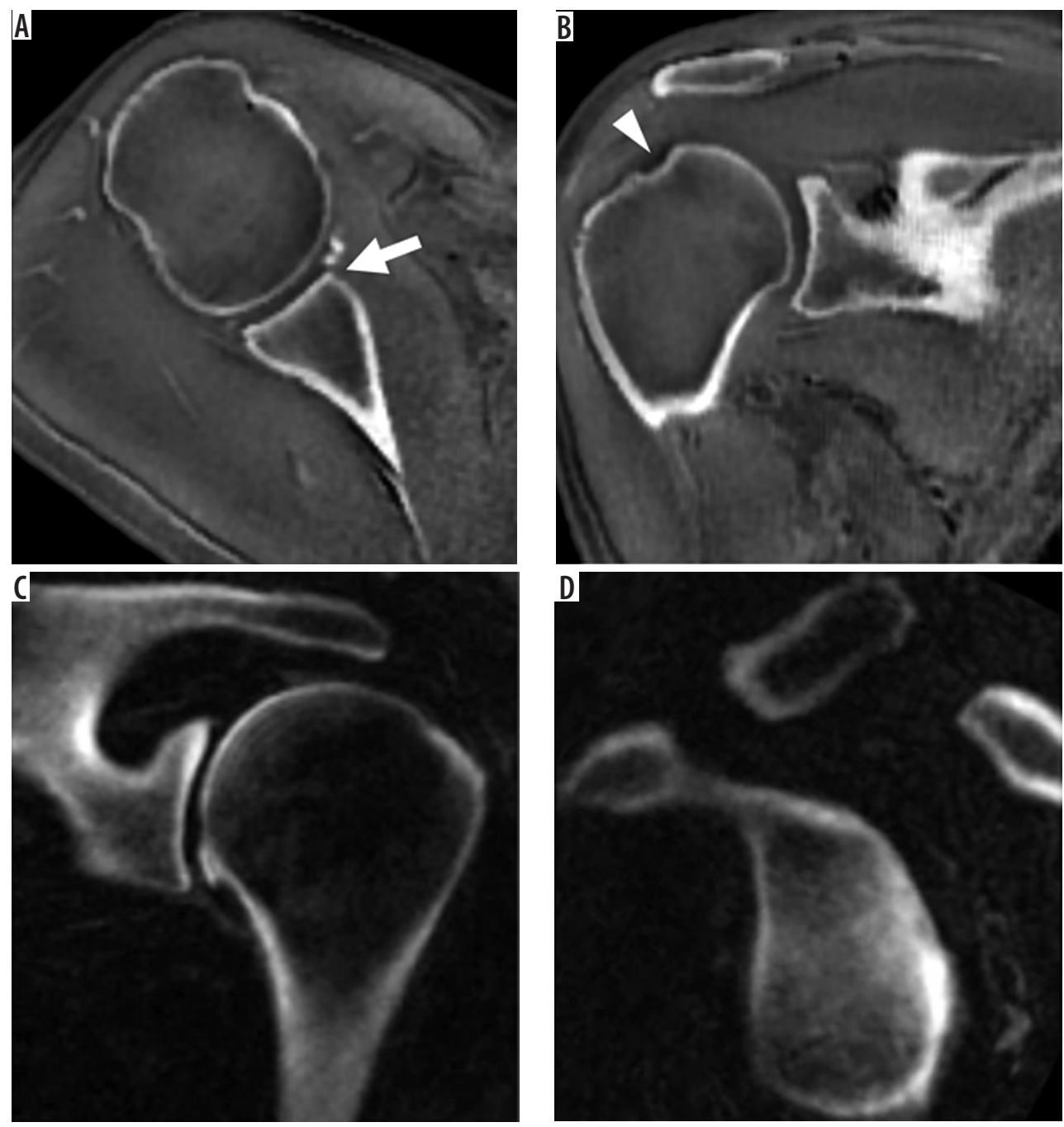

Figure 16. Magnetic resonance (MR) imaging with computed tomography-like contrast in a 48-year-old woman with chronic glenohumeral joint dislocations $(A, B)$ and a shoulder specimen (C, D). $0.8 \mathrm{~mm}^{3}$ isotropic ZTE dataset obtained in 8 minutes scan time allows for multi-planar reconstruction in the (A) axial and (B) coronal oblique planes, and visualisation of a Bankart fracture with small adjacent fragments (arrow) and a Hill-Sachs lesion (arrowhead). IR-UTE MR images in the (A) coronal and (B) sagittal oblique planes show high-contrast bone images with excellent soft tissue suppression 

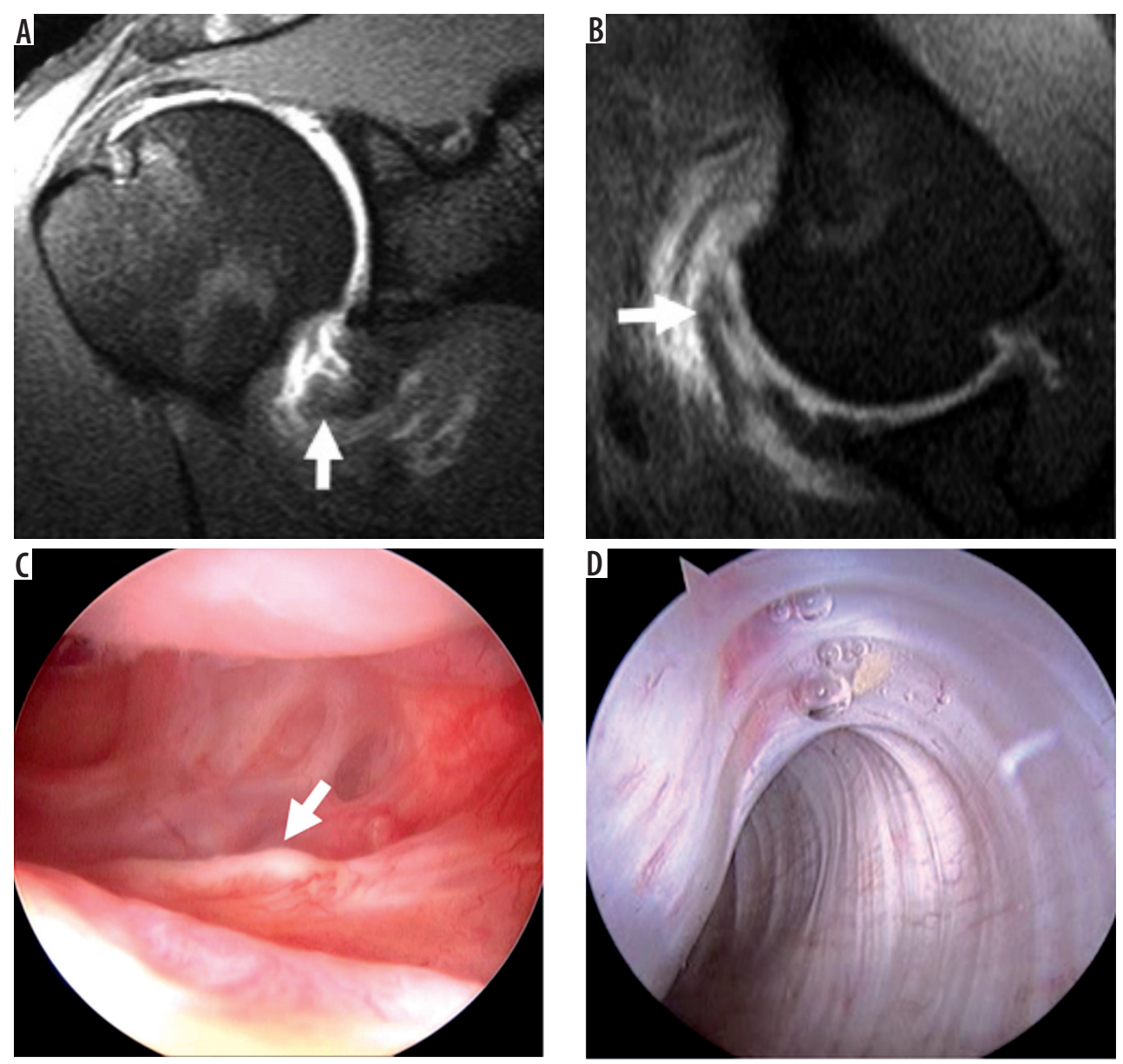

Figure 17. Humeral avulsion of the inferior glenohumeral ligament (HAGL) lesion in a 27-year-old professional pitcher (A-C). A) Coronal oblique and B) ABER T1-weighted, fat-suppressed magnetic resonance arthrogram images show a torn, retracted, and scarred anterior band of the inferior glenohumeral ligament (arrows) with extra-articular contrast extravasation. C) Arthroscopic image confirms the HAGL lesion with the retracted and scarred margin (arrow) and surrounding inflammation. D) Arthroscopic image of an intact IGHL in a 22-year-old for comparison

Table 2. Bankart and Bankart variant lesions associated with anterior glenohumeral instability

\begin{tabular}{|l|l|}
\hline Type & Image findings \\
\hline Bankart & Lesion and detachment of the anteroinferior glenoid labrum \\
\hline Osseous Bankart & Fracture and avulsion of the anteroinferior glenoid, including the glenoid labrum \\
\hline ALPSA & Lesion of the anteroinferior glenoid labrum associated with sleeve avulsion of the scapular periosteum \\
\hline Perthes & Lesion of the anteroinferior glenoid labrum attached to an intact scapular periosteum \\
\hline GLAD & Lesion of the anteroinferior labrum associated with an anteroinferior glenoid articular cartilage injury \\
\hline
\end{tabular}

Table 3. Posterior glenoid and labrum lesions associated with posterior glenohumeral instability

\begin{tabular}{|l|l|}
\hline Type & Image findings \\
\hline Posterior Bankart & Lesion and detachment of the posteroinferior glenoid labrum \\
\hline POLPSA & $\begin{array}{l}\text { Lesion of the posteroinferior glenoid labrum associated with sleeve avulsion of the scapular periosteum which is attached } \\
\text { to the displaced labral tissue }\end{array}$ \\
\hline Bennett & Ossification at the posterior glenoid associated with posterior labral lesion \\
\hline Kim & Lesion of the posterior glenoid labrum associated with posterior glenoid articular cartilage injury \\
\hline
\end{tabular}

inferior labral tear with adjacent cartilage injury. The MR arthrogram images may show a collection of contrast/ fluid between a torn glenoid labrum and the glenoid rim, which is thought to be a specific finding for a labral tear
(Table 2). ABER positioning of the patient is particularly useful in assessing these anterior instability lesions [4]. This can be achieved by having the patient place the arm behind their head. MR arthrography may also show Ban- 

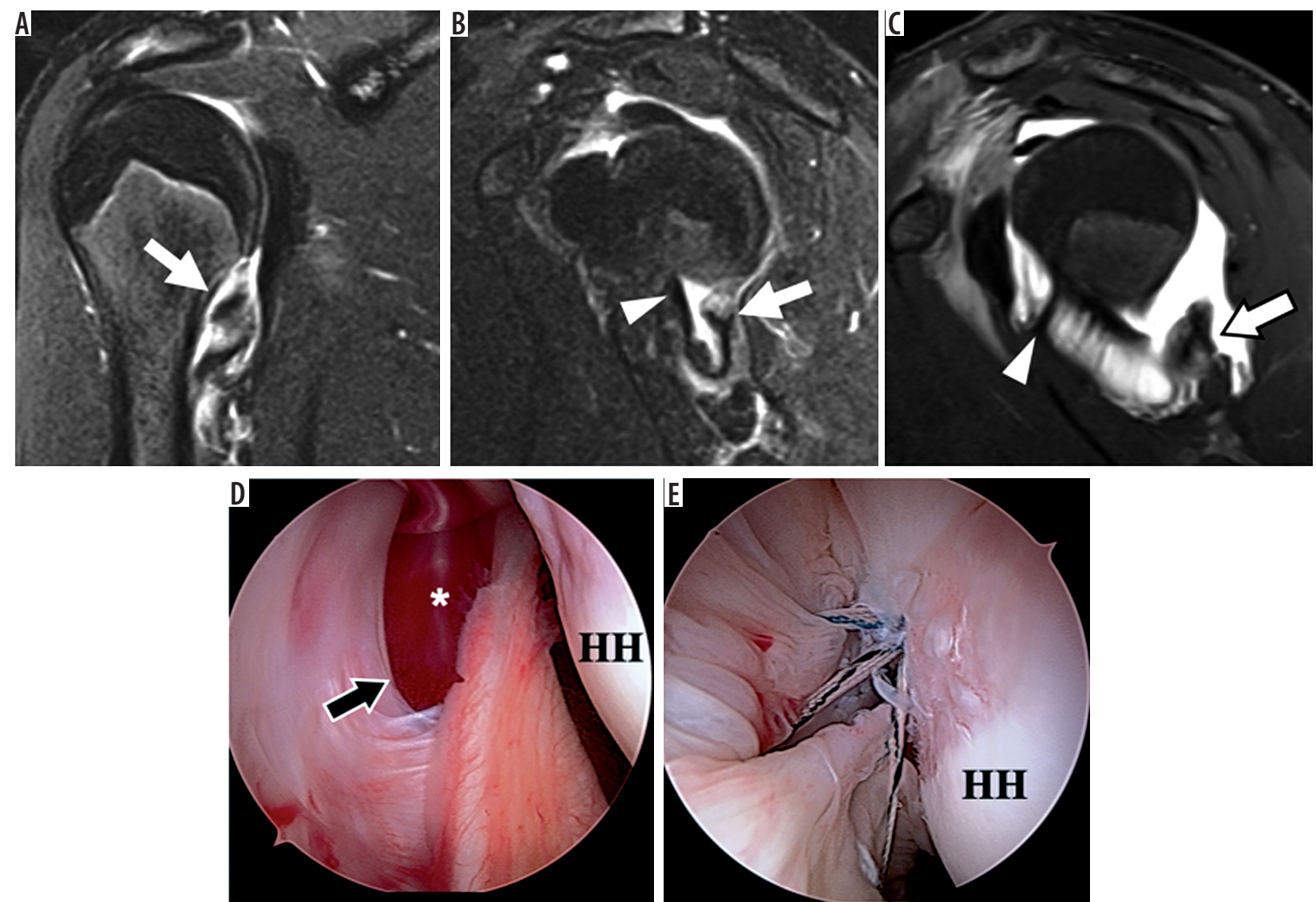

Figure 18. Posterior humeral avulsion of the inferior glenohumeral ligament (HAGL) lesion in a 17-year-old with shoulder dislocation during wrestling match. A) Coronal and B) sagittal oblique, non-contrast, T2-weighted, fat-suppressed MR images show a torn and retracted posterior band of the IGHL (arrows). The anterior band is intact (arrowhead). C) Sagittal, oblique, proton density-weighted, fat-suppressed MR image after intra-articular injection of contrast, obtained 1 month after the non-contrast exam, confirms the intact anterior band (arrowhead) and torn, retracted, and scarred posterior band of the IGHL (arrow). D) Arthroscopic image shows the torn margin of the posterior band (arrow) with the rotator cuff muscle visible posteriorly through the defect (asterisk). E) Arthroscopic image shows the repaired IGHL complex using sutures and an anchor. HH - humeral head
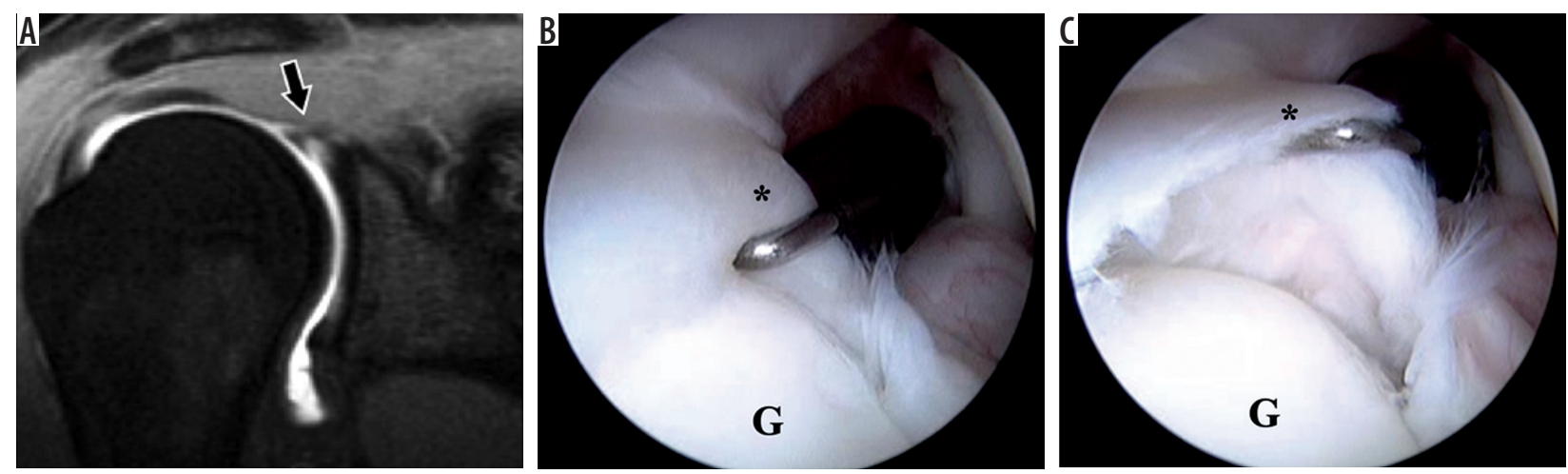

Figure 19. Superior labrum anterior-posterior (SLAP) tear in a 22-year-old professional baseball pitcher with shoulder pain. A) Coronal oblique, T1-weighted, fat-suppressed magnetic resonance arthrography image shows contrast extending into the labral substance, consistent with a SLAP tear. B, C) Arthroscopic images show the unstable, detached superior labrum (asterisk) being uplifted by the probe. $\mathrm{G}$ - glenoid

kart fracture, a torn inferior glenohumeral ligament, bone contusion of the humeral head, and Hill-Sachs fracture. The Bankart fracture fragment may vary in size, and the anteroinferior labrum may be displaced, show an intrasubstance tear, or be abnormally small or absent. A recent study identified preglenoid fat thickness as an ancillary sign on non-contrast MRI for diagnosis of a Bankart le- sion and its variants, with fat tissue thinner than $1.6 \mathrm{~mm}$ showing a significantly increased chance of an abnormality being present. However, this sign showed no significant difference in identifying the Bankart variants from each other [64], which reinforces the clinical importance of MR arthrography for the evaluation of labroligamentous pathology. 

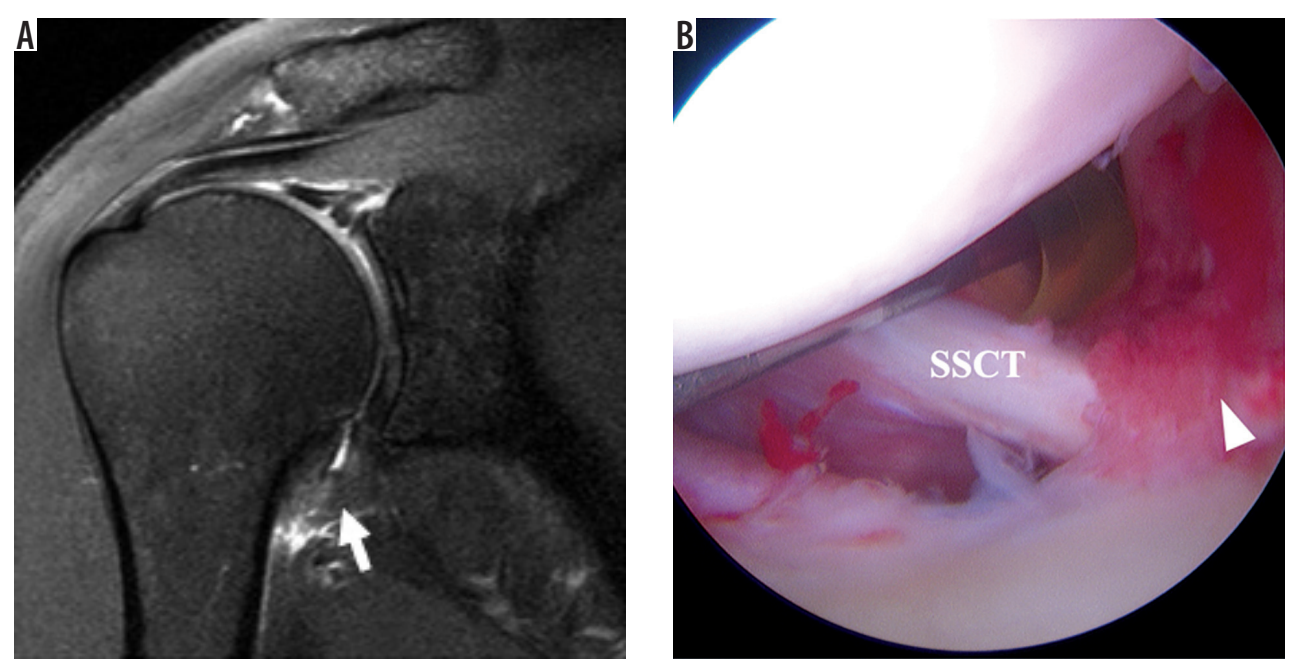

Figure 20. Adhesive capsulitis of the shoulder. A) Coronal oblique, T2-weighted, fat-suppressed magnetic resonance image shows massively thickened and oedematous inferior glenohumeral ligament (IGHL) complex (arrow). B) Arthroscopic image confirms synovitis in the rotator interval (arrowhead). SSCT - subscapularis tendon
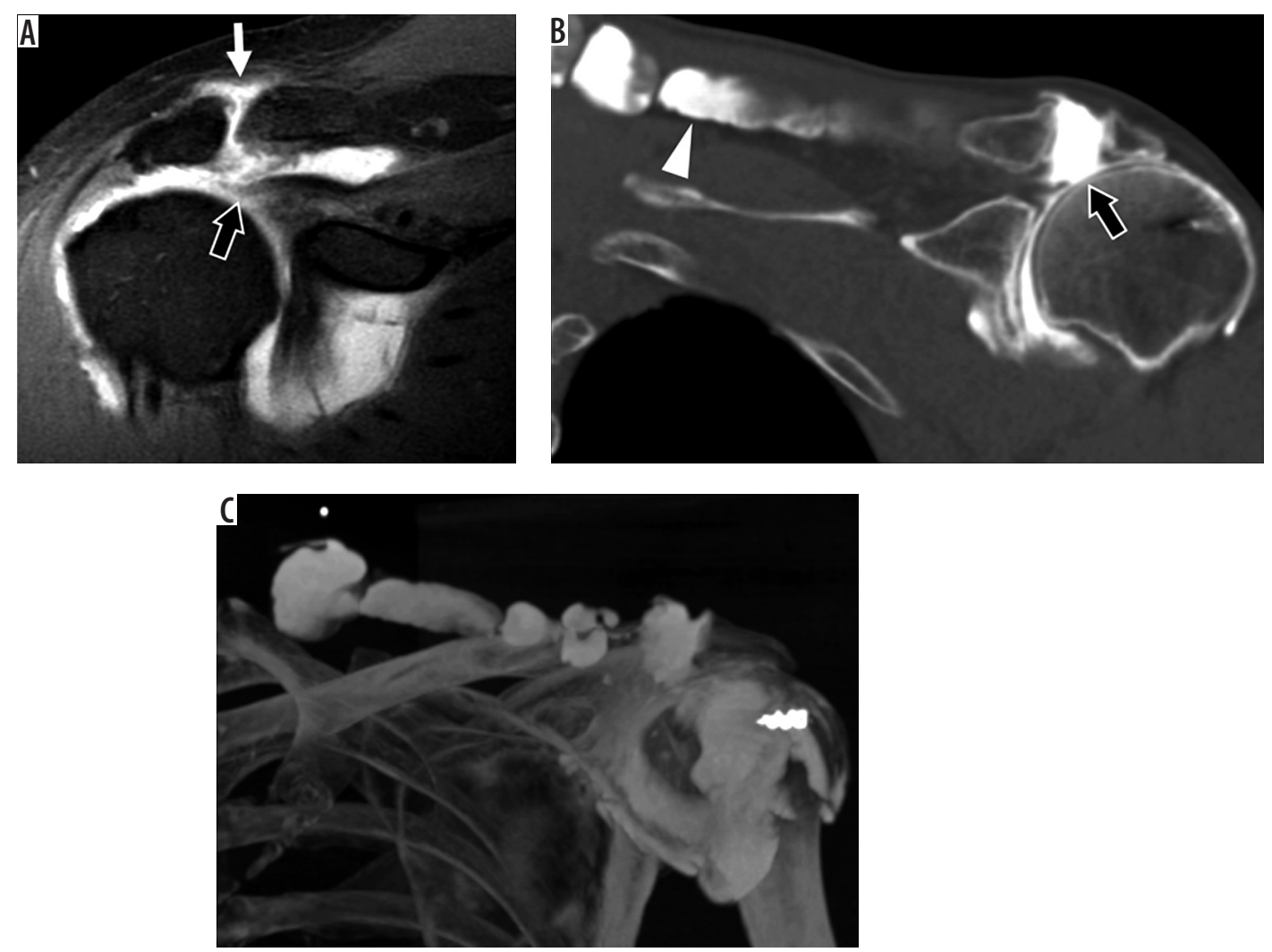

Figure 21. Geyser signs in a 56-year-old man (A) and a 62-year-old man status post cuff repair (B, C). A) Coronal oblique, T1-weighted, fat-suppressed magnetic resonance image post intra-articular contrast administration shows a retracted, full-thickness superior cuff tear (thick arrow) with contrast extending into the AC joint (thin arrow). B) Coronal oblique computed tomography (CT) arthrogram image shows contrast extending through tears of the superior cuff and inferior AC joint capsule (thick arrow), and into the trapezius muscle belly (arrowhead). C) 3D CT maximum intensity projection image shows the full extent of the ganglion cyst. An external marker was used to delineate the mass the patient was feeling

Posterior dislocations of the humeral head are much less common, usually associated with epileptic seizures, and due to internal rotation while the arm is abducted. The posterior band of the inferior glenohumeral ligament may be damaged, leading to recurrent posterior instability. Radiographs can be used for diagnosis, although it helps to have more than an anterior-posterior view. MRI can be helpful in assessing reverse
Hill-Sachs (impaction fracture of the anteromedial humeral head), reverse Bankart (detachment of the posteroinferior labrum), and other reverse Bankart variants like the posterior labrocapsular periosteal sleeve avulsion (POLPSA), lesions of the glenoid articular cartilage associated with a posterior labral lesion (Kim lesion), and chronic ossification of the glenoid labrum (Bennett lesion) [65-67] (Table 3). 
Table 4. Snyder classification of superior labral anteroposterior (SLAP) tear [72]

\begin{tabular}{|l|l|}
\hline Type & Image findings \\
\hline I & Fraying of the superior labrum \\
\hline II & Detachment of the superior labrum and the biceps origin \\
\hline III & Bucket handle tear of the superior labrum with intact biceps origin \\
\hline IV & Bucket handle tear of the superior labrum with extension into biceps tendon \\
\hline
\end{tabular}

Table 5. Maffet classification of labral tear [74]

\begin{tabular}{|l|l|}
\hline Type & Image findings \\
\hline V & Anteroinferior labral lesion extending superiorly and into the biceps tendon \\
\hline VI & Unstable superior labral lesion with biceps tendon detachment \\
\hline VII & Superior labral and biceps tendon lesion extending to middle glenohumeral ligament \\
\hline
\end{tabular}

Table 6. Powell classification of labral tear [96]

\begin{tabular}{|l|l|}
\hline Type & Image findings \\
\hline VIII & Posterior labral lesion extending to the inferior portion of the labrum \\
\hline IX & Labral lesion extending the entire circumference of the glenoid \\
\hline$X$ & Superior and associated posteroinferior labral tear \\
\hline
\end{tabular}

The labral-ligamentous complex may be damaged at a site other than the glenoid or labrum, as seen in a humeral avulsion of the inferior glenohumeral ligament (HAGL) lesion. This injury is more common in young men participating in contact sports. MRI plays an important role because this lesion may be difficult to appreciate during arthroscopy and can cause significant shoulder instability [21]. It is helpful to assess this lesion in the acute setting while there is oedema and haemorrhage. Findings include avulsion of the inferior glenohumeral ligament at the proximal humerus, retraction of the inferior glenohumeral ligament (J-sign), and other common findings seen in anterior dislocations (Figure 17). MR arthrography is particularly helpful, but iatrogenic extravasation of contrast material can be mistaken for an inferior glenohumeral ligament tear. The findings of a torn anterior band, a thickened ligament ( $>3 \mathrm{~mm}$ ), reverse-tapered calibre, and scarred appearance of the torn margins suggest a tear, while isolated disruption of the posterior axillary pouch is found in iatrogenic extravasation [68]. Posterior HAGL may also occur during a shoulder dislocation, where contrast may dissect through a defect in the posterior band of the inferior glenohumeral ligament [69] (Figure 18).

As mentioned before, for the anteroinferior glenoid and labral pathologies, MR arthrography is also particularly helpful and superior to MRI in evaluating capsular and ligamentous pathology because it provides distension and separation of intraarticular structures and is thus the recommended study according to the ACR Appropriateness Criteria [15]. Axial oblique images obtained in the ABER position can add important clinical information as the glenohumeral ligament is pulled taut, creating traction on the anteroinferior labrum, and exposing partially de- tached anterior and anteroinferior labral and ligamentous tears $[70,71]$.

\section{Superior labrum anterior-posterior tears}

A superior labrum anterior-posterior (SLAP) tear is a tear of the superior labrum that extends anteriorly to posteriorly. Snyder et al. first classified the SLAP lesions in four types [72] (Table 4). In type I the superior labrum is frayed, without detachment. In type II there is detachment of both the superior labrum and the biceps tendon origin. In type III there is a bucket handle tear of the superior labrum without biceps tendon detachment. Finally type IV is a bucket handle tear of the superior labrum associated with detachment of the biceps tendon. The Snyder classification was followed later by Maffet and Powell classifications of new labral lesion subtypes, reaching a total of 10 usually accepted today (Tables 5 and 6) [73,74]. Of note, two type-X lesions exist in the literature. In 2000, Beltran et al. described a SLAP lesion that extends into the rotator interval in the form of a lesion of the superior glenohumeral ligament, coracohumeral ligament, capsule, or synovium [73]. SLAP injuries may be seen in patients who fall on an outstretched hand or those who are involved in overhead throwing sports. For example, in baseball players there may be strain at the biceps tendon anchor due to maximum external rotation, resulting in a "peel-back-tear", which is a detachment of the biceps anchor and posterosuperior labrum from the glenoid [44]. High interobserver variability has been observed in the SLAP tear description among radiologists [73]. It is worth noting that SLAP tears have been found on MRI with high frequency in asymptomatic individuals aged 45-60 years 
and in asymptomatic overhead throwing athletes as well, thus the decision to intervene surgically will depend on additional clinical history and physical exam [75-78]. In some cases, posterior SLAP tears can be seen in combination with posterosuperior cuff tears in throwing athletes.

Care must be taken to not confuse SLAP tears with a sublabral foramen or sublabral recess. MR arthrography has shown better diagnostic accuracy of SLAP tears when comparing $3 \mathrm{~T}$ to $1.5 \mathrm{~T}$, and with manoeuvres like longitudinal traction and ABER [79]. On MR arthrography, high signal can be seen extending into the superior labrum and possibly into the biceps tendon. On the axial images, there may be abnormally wide or irregular high signal interposed between the labrum and glenoid. Coronal oblique images are helpful for evaluating SLAP tears and can show a linear signal through the labrum that is vertical or curves laterally into the labral substance as opposed to a sublabral recess that curves medially at the labral-chondral junction (Figure 19).

\section{Adhesive capsulitis}

Adhesive capsulitis of the shoulder is a clinical diagnosis characterised by active and passive limitation of movement of the glenohumeral joint associated with pain [80]. MRI can be helpful in the diagnosis, showing glenohumeral capsular thickening, periarticular oedema, and gadolinium enhancement (Figure 20). Rotator interval fat obliteration has been described as a specific sign, but it should always be correlated with clinical symptoms. Adhesive capsulitis is also commonly associated with RCT pathology. However, the radiologist should be aware that the increased signal within the capsule leads to a higher percentage of falsepositive interpretations [81]. When intra-tendinous signal intensity approaches fluid signal, however, a tear can be confidently diagnosed.

\section{Acromioclavicular joint}

Trauma to the AC joint and later stages of degenerative joint damage are primarily assessed on radiographic studies $[15,82]$. However, MRI can be helpful in evaluating earlier changes in AC joint degeneration, differentiating sprains and ligaments tears, and showing concomitant glenohumeral joint findings that may be present up to $18 \%$ of the time $[83,84]$. AC joint capsular hypertrophy can be seen on T2-weighted images as an intermediate signal intensity with joint distension. This distension can cause fat stripe obliteration of the SA-SD bursa and impingement of the rotator cuff tendon. There may be a cystic distension superior to the AC joint, which shows high signal intensity on T2-weighted images. If there is AC joint effusion present, it will be bright on T2-weighted images. MRI can help reveal late stages of degenerative disease like acromial and clavicular spurs. Studies have shown that MRI findings of AC joint capsular hypertro- phy can be predictive in the pain relief patients may obtain from intraarticular injections [85]. Studies have noted that although AC joint osteoarthritis is a common finding on MRI, most cases remain asymptomatic and do not require intervention [86].

In the setting of a rotator cuff tear and $\mathrm{AC}$ joint arthrosis, fluid may migrate from the glenohumeral joint, through the SA-SD bursa, and into the AC joint. This is known as the geyser sign and masses can result, either above the AC joint or into the adjacent muscle (Figure 21). If large enough, these masses may mimic a tumour on clinical assessment [87]. MRI can be an important diagnostic tool in these instances, and allow for concomitant evaluation of the AC joint, glenohumeral joint, and rotator cuff pathology $[88,89]$.

\section{Bursae}

Of the multiple shoulder bursae, the SA-SD bursa is one that is frequently involved in shoulder pathology. Most pathological cases are either due to bursitis or secondary involvement in other diseases like rotator cuff tears and tendinopathy, impingement, AC joint pathology, rheumatoid arthritis, or pigmented villonodular synovitis. The presence of SA-SD bursitis has been found to be a contributing factor to pain both in operated patients and the general population [90]. The SA-SD bursa is not directly visualised on radiographs, but instead can be indirectly evaluated by looking at the peribursal fat plane. Ultrasound allows for direct evaluation of the bursae and is excellent for image-guided interventions, which can be superior to blind injections in terms of accuracy [16,91]. Although MRI is superior in evaluating soft-tissue structures and nicely depicts the bursae of the shoulder, it is not usually ordered as a first-line modality in patients with suspected bursitis [16]. The normal SA-SD bursa is one that is $<2 \mathrm{~mm}$ in thickness. Those with $>3 \mathrm{~mm}$ thickness, extension of fluid to the AC joint or glenohumeral joint, and thickening of the bursal wall are thought to be more predictive signs of bursitis [92,93]. When the SA-SD bursa is distended, it may be associated with full-thickness (Figure 3) or partial (Figures 4 and 5) rotator cuff tears [94]. Although some studies have found rotator cuff and bursae abnormalities in asymptomatic individuals and consider them weakly related to symptoms, a study by Kvalvaag et al. found the MRI findings of tendinosis and bursitis to be associated with poorer outcome after conservative treatment [95].

\section{Conclusions}

The high prevalence of shoulder pain is frequently due to internal derangements of the shoulder, and MRI is the preferred modality for evaluation of these conditions. The understanding of these diseases, optimal imaging techniques for evaluation, normal anatomic variants, ab- 
normal MRI findings, diagnostic pitfalls, and limitations of MRI are significant for the diagnosis and treatment of patients. New advances and research in MRI have provided additional potential uses for evaluating shoulder derangements.

\section{Conflict of interest}

The authors report no conflict of interest.

\section{References}

1. Van der Windt DA, Koes BW, de Jong BA, et al. Shoulder disorders in general practice: incidence, patient characteristics, and management. Ann Rheum Dis 1995; 54 : 959-964.

2. Luime JJ, Koes BW, Hendriksen IJ, et al. Prevalence and incidence of shoulder pain in the general population; a systematic review. Scand J Rheumatol 2004; 33: 73-81.

3. Davis SJ, Teresi LM, Bradley WG, et al. Effect of arm rotation on MR imaging of the rotator cuff. Radiology 1991; 181: 265-268.

4. Chung CB ea. ACR-SPR-SSR Practice Parameter for the Performance and Interpretation of Magnetic Resonance Imaging (MRI) of the Shoulder. Available at: https://www.acr.org/-/media/ACR/Files/ Practice-Parameters/MR-Shldr.pdf?la=en. Published Revised 2015 (Accessed: 03.02.2020).

5. McMonagle JS VE. MRI of the shoulder: rotator cuff. Published 2012 (Accessed: 06.02.2020).

6. Bunnell KM, Hemke R, Husseini JS, et al. Does MR arthrography cause intracranial gadolinium deposition? Skeletal Radiol 2020; 49: 1051-1056.

7. Kralik SF, Singhal KK, Frank MS, et al. Evaluation of gadolinium deposition in the brain after MR arthrography. AJR Am J Roentgenol 2018; 211: 1063-1067.

8. Andreisek G, Froehlich JM, Hodler J, et al. Direct MR arthrography at 1.5 and 3.0 T: signal dependence on gadolinium and iodine concentrations-phantom study. Radiology 2008; 247: 706-716.

9. Montgomery DD, Morrison WB, Schweitzer ME, et al. Effects of iodinated contrast and field strength on gadolinium enhancement: implications for direct MR arthrography. J Magn Reson Imaging 2002; 15: 334-343.

10. Resnick DL, Kang HS, Pretterklieber ML. Internal derangements of joints: 2 volume set. Elsevier Canada, Toronto 2006.

11. De Maeseneer M, Van Roy P, Shahabpour M. Normal MR imaging anatomy of the rotator cuff tendons, glenoid fossa, labrum, and ligaments of the shoulder. Radiol Clin North Am 2006; 44: 479-487.

12. Schraner AB, Major NM. MR imaging of the subcoracoid bursa. AJR Am J Roentgenol 1999; 172: 1567-1571.

13. Cadogan A, Laslett M, Hing WA, et al. A prospective study of shoulder pain in primary care: prevalence of imaged pathology and response to guided diagnostic blocks. BMC Musculoskelet Disord 2011; $12: 119$.

14. Piper CC, Hughes AJ, Ma Y, et al. Operative versus nonoperative treatment for the management of full-thickness rotator cuff tears: a systematic review and meta-analysis. J Shoulder Elbow Surg 2018; 27: 572-576.

15. Amini B, Beckmann NM, Beaman FD, et al. ACR appropriateness criteria ${ }^{\circledast}$ shoulder pain-traumatic. J Am Coll Radiol 2018; 15: S171-S188.
16. Small KM, Adler RS, Shah Sh, et al. ACR appropriateness criteria ${ }^{\star}$ shoulder pain-atraumatic. J Am Coll Radiol 2018; 15: S388-S402.

17. Littlewood C, May S, Walters S. Epidemiology of rotator cuff tendinopathy: a systematic review. Shoulder Elbow 2013; 5: 256-265.

18. Neumann CH, Holt RG, Steinbach LS, et al. MR imaging of the shoulder: appearance of the supraspinatus tendon in asymptomatic volunteers. AJR Am J Roentgenol 1992; 158: 1281-1287.

19. Chang EY, Szeverenyi NM, Statum S, et al. Rotator cuff tendon ultrastructure assessment with reduced-orientation dipolar anisotropy fiber imaging. AJR Am J Roentgenol 2014; 202: W376-378.

20. Kalia V, Freehill MT, Miller BS, et al. Multimodality imaging review of normal appearance and complications of the postoperative rotator cuff. AJR Am J Roentgenol 2018; 211: 538-547.

21. Chaipat L, Palmer WE. Shoulder magnetic resonance imaging. Clin Sports Med 2006; 25: 371-386.

22. Khoschnau S, Milosavjevic J, Sahlstedt B, et al. High prevalence of rotator cuff tears in a population who never sought for shoulder problems: a clinical, ultrasonographic and radiographic screening study. Eur J Orthop Surg Traumatol 2020; 30: 457-463.

23. Sharma G, Bhandary S, Khandige G, et al. MR imaging of rotator cuff tears: correlation with arthroscopy. J Clin Diagn Res 2017; 11: TC24-TC27.

24. Zanetti M, Gerber C, Hodler J. Quantitative assessment of the muscles of the rotator cuff with magnetic resonance imaging. Invest Radiol 1998; 33: 163-170.

25. Naimark M, Trinh T, Robbins C, et al. Effect of muscle quality on operative and nonoperative treatment of rotator cuff tears. Orthop J Sports Med 2019; 7: 2325967119863010.

26. Carvalho CD, Cohen C, Belangero PS, et al. Partial rotator cuff injury in athletes: bursal or articular? Rev Bras Ortop 2015; 50: 416-421.

27. Nakajima T, Rokuuma N, Hamada K, et al. Histologic and biomechanical characteristics of the supraspinatus tendon: Reference to rotator cuff tearing. J Shoulder Elbow Surg 1994; 3: 79-87.

28. Jeong S, Choi JY, Kang YS, et al. Disproportionate fluid sign as an aid in diagnosing high-grade bursal-sided supraspinatus tendon tear. Acta Radiol 2018; 59: 1102-1109.

29. Chang EY, Chung CB. Current concepts on imaging diagnosis of rotator cuff disease. Semin Musculoskelet Radiol 2014; 18: 412-424.

30. Mesiha MM, Derwin KA, Sibole SC, et al. The biomechanical relevance of anterior rotator cuff cable tears in a cadaveric shoulder model. J Bone Joint Surg Am 2013; 95: 1817-1824.

31. Huri G, Kaymakoglu M, Garbis N. Rotator cable and rotator interval: anatomy, biomechanics and clinical importance. EFORT Open Rev 2019; 4: 56-62.

32. Sonnabend DH, Watson EM. Structural factors affecting the outcome of rotator cuff repair. J Shoulder Elbow Surg 2002; 11: 212218. 
33. Kwon J, Lee YH, Kim SH, et al. Delamination does not affect outcomes after arthroscopic rotator cuff repair as compared with nondelaminated rotator cuff tears: a study of 1043 consecutive cases. Am J Sports Med 2019; 47: 674-681.

34. Lunn JV, Castellanos-Rosas J, Tavernier T, et al. A novel lesion of the infraspinatus characterized by musculotendinous disruption, edema, and late fatty infiltration. J Shoulder Elbow Surg 2008; 17: 546-553.

35. Huang BK, Chang EY. Delaminating infraspinatus tendon tears with differential retraction: imaging features and surgical relevance. Skeletal Radiol 2017; 46: 41-50.

36. Huang T, Liu J, Ma Y, et al. Diagnostic accuracy of MRA and MRI for the bursal-sided partial-thickness rotator cuff tears: a metaanalysis. J Orthop Surg Res 2019; 14: 436.

37. Singer AD, Rosenthal J, Umpierrez M, et al. A comparison of saline and gadolinium shoulder MR arthrography to arthroscopy. Skeletal Radiol 2020; 49: 625-633.

38. Michener LA, McClure PW, Karduna AR. Anatomical and biomechanical mechanisms of subacromial impingement syndrome. Clin Biomech 2003; 18: 369-379.

39. Kenmoku T, Matsuki K, Ochiai N, et al. Comparison of glenohumeral joint rotation between asymptomatic subjects and patients with subacromial impingement syndrome using cine-magnetic resonance imaging: a cross-sectional study. BMC Musculoskelet Disord 2019; 20: 475 .

40. Bencardino JT. The shoulder. Imaging diagnosis with clinical implications. Springer International Publishing, Berlin 2019.

41. Vanarthos WJ, Monu JU. Type 4 acromion: a new classification. Contemp Orthop 1995; 30: 227-229.

42. Chang EY, Moses DA, Babb JS, et al. Shoulder impingement: objective $3 \mathrm{~d}$ shape analysis of acromial morphologic features. Radiology 2006; 239: 497-505.

43. Heyworth BE, Williams RJ, 3rd. Internal impingement of the shoulder. Am J Sports Med 2009; 37: 1024-1037.

44. Lin DJ, Wong TT, Kazam JK. Shoulder injuries in the overheadthrowing athlete: epidemiology, mechanisms of injury, and imaging findings. Radiology 2018; 286: 370-387.

45. Tarkowski EM, Omar IM, Blount KJ, et al. Subscapularis myotendinous junction tears presenting with posterior shoulder pain in overhead throwing athletes. Acta Med Acad 2019; 48: 205-216.

46. Hayes CW, Conway WF. Calcium hydroxyapatite deposition disease. Radiographics 1990; 10: 1031-1048.

47. Flemming DJ, Murphey MD, Shekitka KM, et al. Osseous involvement in calcific tendinitis: a retrospective review of 50 cases. AJR Am J Roentgenol 2003; 181: 965-972.

48. Pereira BP, Chang EY, Resnick DL, et al. Intramuscular migration of calcium hydroxyapatite crystal deposits involving the rotator cuff tendons of the shoulder: report of 11 patients. Skeletal Radiol 2016; 45: $97-103$

49. Ma YJ, Shao H, Du J, et al. Ultrashort echo time magnetization transfer (UTE-MT) imaging and modeling: magic angle independent biomarkers of tissue properties. NMR Biomed 2016; 29: 1546-1552.

50. Zhu Y, Cheng X, Ma Y, et al. Rotator cuff tendon assessment using magic-angle insensitive 3D ultrashort echo time cones magnetization transfer (UTE-Cones-MT) imaging and modeling with histological correlation. J Magn Reson Imaging 2018; 48: 160-168.
51. Ashir A, Ma Y, Jerban S, et al. Rotator cuff tendon assessment in symptomatic and control groups using quantitative MRI. J Magn Reson Imaging 2020. doi: 10.1002/jmri.27115.

52. Lazik-Palm A, Kraff O, Rietsch SHG, et al. 7-T clinical MRI of the shoulder in patients with suspected lesions of the rotator cuff. Eur Radiol Exp 2020; 4: 10.

53. Coninck TD, Ngai SS, Tafur M, et al. Imaging the glenoid labrum and labral tears. RadioGraphics 2016; 36: 1628-1647.

54. Davidson PA, Rivenburgh DW. Mobile superior glenoid labrum: a normal variant or pathologic condition? Am J Sports Med 2004; 32: 962 966.

55. Kadi R, Milants A, Shahabpour M. Shoulder Anatomy and Normal Variants. J Belg Soc Radiol 2017; 101(Suppl 2): 3.

56. Eshoj H, Rasmussen S, Frich LH, et al. A neuromuscular exercise programme versus standard care for patients with traumatic anterior shoulder instability: study protocol for a randomised controlled trial (the SINEX study). Trials 2017; 18: 90.

57. Orvets ND, Parisien RL, Curry EJ, et al. Acute Versus Delayed Magnetic Resonance Imaging and Associated Abnormalities in Traumatic Anterior Shoulder Dislocations. Orthop J Sports Med 2017; 5: 2325967117728019.

58. Hirai Gimber L, Taljanovic MS, Rockov ZA, et al. The veil of obscuration: additional radiographic sign of posterior shoulder dislocation. Acta Med Acad 2018; 47: 165-175.

59. Gyftopoulos S, Beltran LS, Bookman J, et al. MRI evaluation of bipolar bone loss using the on-track off-track method: a feasibility study. Am J Roentgenol 2015; 205: 848-852.

60. Di Giacomo G, de Vita A, Costantini A, et al. Management of humeral head deficiencies and glenoid track. Curr Rev Musculoskelet Med 2014; 7: 6-11.

61. Breighner RE, Endo Y, Konin GP, et al. Technical developments: zero echo time imaging of the shoulder: enhanced osseous detail by using MR imaging. Radiology 2018; 286: 960-966.

62. Ma YJ, West J, Nazaran A, et al. Feasibility of using an inversion-recovery ultrashort echo time (UTE) sequence for quantification of glenoid bone loss. Skeletal Radiol 2018; 47: 973-980.

63. Richards RD, Sartoris DJ, Pathria MN, et al. Hill-Sachs lesion and normal humeral groove: MR imaging features allowing their differentiation. Radiology 1994; 190: 665-668.

64. Polat G, Ogul H, Yalcin A, et al. An ancillary sign in the diagnosis of bankart and bankart variants in patients with glenohumeral instability on nonarthrographic magnetic resonance imaging: preglenoid fat thickness. J Comput Assist Tomogr 2019; 43: 367-371.

65. Yu JS, Ashman CJ, Jones G. The POLPSA lesion: MR imaging findings with arthroscopic correlation in patients with posterior instability. Skeletal Radiol 2002; 31: 396-399.

66. Chung CB, Sorenson S, Dwek JR, et al. Humeral avulsion of the posterior band of the inferior glenohumeral ligament: MR arthrography and clinical correlation in 17 patients. Am J Roentgenol. 2004; 183: 355-359.

67. Ferrari JD, Ferrari DA, Coumas J, et al. Posterior ossification of the shoulder: the Bennett lesion. Etiology, diagnosis, and treatment. Am J Sports Med 1994; 22: 171-176.

68. Wang W, Huang BK, Sharp M, et al. MR arthrogram features that can be used to distinguish between true inferior glenohumeral ligament complex tears and iatrogenic extravasation. AJR Am J Roentgenol 2019; 212: 411-417. 
69. Demehri S, Hafezi-Nejad N, Fishman EK. Advanced imaging of glenohumeral instability: the role of MRI and MDCT in providing what clinicians need to know. Emerg Radiol 2017; 24: 95-103.

70. Tirman PF, Bost FW, Steinbach LS, et al. MR arthrographic depiction of tears of the rotator cuff: benefit of abduction and external rotation of the arm. Radiology 1994; 192: 851-856.

71. Saleem AM, Lee JK, Novak LM. Usefulness of the abduction and external rotation views in shoulder MR arthrography. Am J Roentgenol 2008; 191: 1024-1030.

72. Snyder SJ, Karzel RP, del Pizzo W, et al. SLAP lesions of the shoulder. Arthroscopy 1990; 6: 274-279.

73. Chang EY, Fliszar E, Chung CB. Superior labrum anterior and posterior lesions and microinstability. Magn Reson Imaging Clin N Am 2012; 20: 277-294.

74. Maffet MW, Gartsman GM, Moseley B. Superior labrum-biceps tendon complex lesions of the shoulder. Am J Sports Med 1995; 23: 93-98.

75. Schwartzberg R, Reuss BL, Burkhart BG, et al. High prevalence of superior labral tears diagnosed by MRI in middle-aged patients with asymptomatic shoulders. Orthop J Sports Med 2016; 4 : 2325967115623212.

76. Miniaci A, Mascia AT, Salonen DC, et al. Magnetic resonance imaging of the shoulder in asymptomatic professional baseball pitchers. Am J Sports Med 2002; 30: 66-73.

77. Connor PM, Banks DM, Tyson AB, et al. Magnetic resonance imaging of the asymptomatic shoulder of overhead athletes: a 5-year follow-up study. Am J Sports Med 2003; 31: 724-727.

78. Chalmers PN, Verma NN. Proximal biceps in overhead athletes. Clin Sports Med 2016; 35: 163-179.

79. Boutin RD, Marder RA. MR Imaging of SLAP lesions. Open Orthop J 2018; $12: 314-323$

80. Zappia M, di Pietto F, Aliprandi A, et al. Multi-modal imaging of adhesive capsulitis of the shoulder. Insights Imaging 2016; 7: 365-371.

81. Loeffler BJ, Brown SL, d'Alessandro DF, et al. Incidence of false positive rotator cuff pathology in MRIs of patients with adhesive capsulitis. Orthopedics 2011; 34: 362.

82. Pogorzelski J, Beitzel K, Ranuccio F, et al. The acutely injured acromioclavicular joint - which imaging modalities should be used for accurate diagnosis? A systematic review. BMC Musculoskelet Disord 2017; 18: 515

83. Tischer T, Salzmann GM, El-Azab H, et al. Incidence of associated injuries with acute acromioclavicular joint dislocations types III through V. Am J Sports Med 2009; 37: 136-139.

84. Nemec U, Oberleitner G, Nemec SF, et al. MRI versus radiography of acromioclavicular joint dislocation. AJR Am J Roentgenol 2011; 197: 968-973.

85. Strobel K, Pfirrmann CW, Zanetti M, et al. MRI features of the acromioclavicular joint that predict pain relief from intraarticular injection. AJR Am J Roentgenol 2003; 181: 755-760.

86. Frigg A, Song D, Willi J, et al. Seven-year course of asymptomatic acromioclavicular osteoarthritis diagnosed by MRI. J Shoulder Elbow Surg 2019; 28: e344-e351.

87. Motamedi D, Everist BM, Mahanty SR, et al. Pitfalls in shoulder MRI: part 2-biceps tendon, bursae and cysts, incidental and postsurgical findings, and artifacts. AJR Am J Roentgenol 2014; 203: 508-515.
88. Maziak N, Plachel F, Scheibel M, et al. Acromioclavicular joint cyst formation in a patient with rotator cuff-tear arthropathy: a rare cause of shoulder discomfort. BMJ Case Rep 2018; 2018: bcr2018226188.

89. Cooper HJ, Milillo R, Klein DA, et al. The MRI geyser sign: acromioclavicular joint cysts in the setting of a chronic rotator cuff tear. Am J Orthop (Belle Mead NJ) 2011; 40: E118-121.

90. Draghi F, Scudeller L, Draghi AG, et al. Prevalence of subacromialsubdeltoid bursitis in shoulder pain: an ultrasonographic study. J Ultrasound 2015; 18: 151-158.

91. Wu T, Song HX, Dong Y, et al. Ultrasound-guided versus blind subacromial-subdeltoid bursa injection in adults with shoulder pain: a systematic review and meta-analysis. Semin Arthritis Rheum 2015; 45: 374-378.

92. Allen H, Chan BY, Davis KW, et al. Overuse injuries of the shoulder. Radiol Clin North Am 2019; 57: 897-909.

93. White EA, Schweitzer ME, Haims AH. Range of normal and abnormal subacromial/subdeltoid bursa fluid. J Comput Assist Tomogr 2006; 30: 316-320.

94. Vahlensieck M. MRI of the shoulder. Eur Radiol 2000; 10: 242-249.

95. Kvalvaag E, Anvar M, Karlberg AC, et al. Shoulder MRI features with clinical correlations in subacromial pain syndrome: a cross-sectional and prognostic study. BMC Musculoskelet Disord 2017; 18: 469.

96. Powell SE, Nord KD, Ryu RKN. The diagnosis, classification, and treatment of SLAP lesions. Oper Tech Sports Med 2004; 12: 99-110. 